\title{
Opportunistic Spectrum Sharing Schemes for CDMA-Based Uplink MAC in Cognitive Radio Networks
}

\author{
Xi Zhang, Senior Member, IEEE, and Hang Su, Student Member, IEEE
}

\begin{abstract}
We consider a wireless cognitive radio network in which a set of secondary users (SUs) opportunistically utilize the wireless spectrum licensed to the primary users (PUs) to transmit packets to the secondary base station (SBS). It is challenging to maximize the spectrum utilization while limiting the interference imposed to PUs due to SUs. To achieve the optimal tradeoff between the spectrum utilization and the interference caused by SUs, we propose the adaptive spectrum sharing schemes for code division multiple access (CDMA) based cognitive medium access control (MAC) in the uplink communications over the cognitive radio networks. Our proposed schemes address the joint problems of channel sensing, data transmission, and power and rate allocations. Under our proposed schemes, the SUs can adaptively select between the intrusive spectrum sharing and the non-intrusive spectrum sharing operations to transmit data to SBS based on the channel utilization, traffic load, and interference constraints. Our proposed schemes enable the SUs to efficiently utilize the available frequency spectrum which is licensed to the PUs while stringently limiting the interference to the PUs. Also conducted are extensive simulations to validate and evaluate our proposed schemes, which show the superiority of our proposed schemes as compared with the other schemes.
\end{abstract}

Index Terms-Cognitive radio networks, code division multiple access (CDMA), power control, dynamic spectrum access (DSA), decision process.

\section{INTRODUCTION}

$\mathbf{T}$ HE TRADITIONAL fixed spectrum allocation method in which the spectrum bands are assigned statically to wireless services results in the spectral under-utilization. As it is reported in recent literature, there is a large amount of spectrum band that is under-utilized [1]. This motivates the concept of dynamic spectrum access (DSA) that allows secondary users (SUs) in the secondary cognitive radio networks to opportunistically use the vacant spectrum which is not being used by the primary users (PUs). On one hand, by allowing opportunistic spectrum access, the overall spectrum utilization can be improved. On the other hand, transmissions by SUs inevitably introduce harmful interference to PUs. Therefore, one of the most critical design criteria for cognitive radio networks is to maintain a balance between the two

Manuscript received 1 December 2009; revised 1 June 2010 and 30 June 2010. The research reported in this paper was supported in part by the National Science Foundation CAREER Award under Grant ECS-0348694.

The authors are with the Networking and Information Systems Laboratory, Department of Electrical and Computer Engineering, Texas A\&M University, College Station, TX 77843 USA (e-mails: xizhang@ece.tamu.edu; su@tamu.edu).

Digital Object Identifier 10.1109/JSAC.2011.110405. contradictory goals: i) minimizing the interference imposed to the PUs and ii) maximizing the overall spectrum utilization.

According to the relationship between the PUs' and SUs' signals, the approaches to implement the cognitive radio networks can be categorized into three types, namely overlay, underlay, and interweave [2]. For the overlay approach (e.g., [3]), the PUs' and SUs' signals can co-exist at the same frequency band simultaneously. The key idea of the overlay approach is that SUs may use a part of their energy to assist the communications of PUs through cooperative communications techniques and the rest of the energy to transmit their own signals. As a result, the interference from the SUs' signals can be compensated with the gain for the PUs' signal quality through the cooperation of the SUs. However, the implementation of the overlay approach requires that the SUs know the PUs' packets before the PUs begin their transmissions, which is not practical in the realistic cognitive radio networks.

Thus, in this paper we mainly focus on the rest two approaches. First, in the underlay approach the PUs' and SUs' signals can also co-exist at the same frequency band simultaneously. In particular, the SUs can "share" the licensed spectrum with the PUs simultaneously by using spread spectrum techniques, such as ultrawide band (UWB) and code division multiple access (CDMA). Thus, the SUs transmit signals in such a low power level that the interference caused by the SUs is below the noise floor of the spectrum. In other words, in the view point of PUs, the transmissions by SUs are nothing but noise with the low-level power. Since the PU cannot perceive the existence of SUs, we also call the underlay approach as the non-intrusive spectrum sharing scheme.

Second, in the interweave approach the SUs opportunistically exploit the spectral holes to communicate. Specifically, the SUs frequently sense the channel. When the SUs discover that the PUs are absent from the licensed spectrum, the SUs do not need to worry about the interference temperature constraint $^{1}$ of PUs and can significantly relax the power constraints imposed onto SUs as compared with the underlay approach. In other words, the SUs can fully take over the licensed spectrum, and thus adopt any transmit power level which is not limited by the PUs. For the ideal case where the SUs can be synchronized with the PUs and the perfect

\footnotetext{
${ }^{1}$ The interference temperature constraint is defined as the maximum acceptable level of RF interference at the receiver antenna in the frequency band of interest. In other words, any transmission is considered to be harmful if it increases the noise floor above the interference-temperature constraint.
} 
sensing can be achieved, SUs do not impose any interference to PUs. However, due to half-duplex of the wireless radio, SUs cannot sense the channel while transmitting their own signals simultaneously, which implies that the SUs cannot accurately know when the PUs become active again, especially when the SUs are not synchronized with PUs. Therefore, the SUs may result in the scenarios where the PUs cannot correctly transmit/receive signals for a certain period, which significantly impairs the performance of PUs. In this sense, we call the interweave approach as the intrusive spectrum sharing scheme.

It is clear that there exist both advantages and disadvantages for the intrusive and non-intrusive spectrum sharing schemes, respectively. In particular, the non-intrusive spectrum sharing scheme is more conservative than the intrusive spectrum sharing scheme in terms of power constraints, and thus causes the less interference to the PUs. On the other hand, the intrusive spectrum sharing scheme can utilize the entire vacant spectrum without considering any interference temperature constraint for PUs, if the channel is sensed and found to be idle. However, when the SUs perform the channel sensing, all of the SUs should keep their radios silent in order to obtain an accurate sensing outcome. During this silent period, no transmissions can be made, which implies that the channel sensing is an overhead for the system. The sensing overhead is considerable, especially when the more accurate sensing is required. Moreover, the intrusive spectrum sharing scheme may cause the direct interference to PUs if the PUs become active and re-utilize their spectrum when SUs are transmitting.

To achieve the optimal tradeoff between the interference imposed onto the PUs and the overall spectrum utilization, in this paper we propose the opportunistic spectrum sharing schemes for the adaptive CDMA medium access control (MAC) in the uplink cognitive radio networks, which combines the intrusive and non-intrusive spectrum sharing schemes together. In particular, under our proposed schemes, the SUs can adaptively select either the intrusive spectrum sharing or non-intrusive spectrum sharing schemes to transmit data based on the channel utilization, traffic load, and interference constraints. Our proposed schemes take into consideration the joint channel sensing and data transmissions, and power and rate allocations.

The rest of this paper is organized as follows. Section II discusses the related work. Section III describes the system models. Section IV addresses power and rate allocations of CDMA uplink communications and develops our proposed schemes. Section V evaluates the performance of our proposed schemes. The paper concludes with Section VI.

\section{THE RELATED WORKS}

The cognitive radio technology has received extensive attention since it was first conceived in 2000 [4]. The cognitive radio is typically built up on top of the software-defined radio (SDR) technology, in which the transmitter's operating parameters, such as frequency range, modulation type, and maximum transmit power can be altered by software [5][7]. There has been already a large body of literature in the interweave (intrusive) and underlay (non-intrusive) spectrum sharing approaches. The interweave based joint transmission and sensing schemes for the much simpler cases of the timeslotted cognitive radio networks can be found in [8]-[10].

For the non-time-slotted cognitive radio networks, the authors of [11] proposed a cognitive MAC protocol aiming to opportunistically utilize the TV broadcast bands. In [12], we developed an interference-duration constrained cognitive MAC protocol for the asynchronous cognitive radio networks. The authors of [13] proposed a underlay-based opportunistic spectrum accessing scheme, which limits the power of secondary signal to satisfy the QoS constraints of the PUs. The authors of [14] studied the underlay approach by formulating a dynamic game where the strategy of a SU is selected solely based on the pricing information. However, the aforementioned works utilize either the intrusive-spectrum sharing scheme alone or the non-intrusive spectrum sharing scheme only, each of which alone cannot high-efficiently utilize the spectrum. Our schemes proposed in this paper integrate the intrusive and non-intrusive spectrum sharing schemes together, and adaptively select the spectrum sharing modes based on the channel utilization, traffic load, and interference constraints.

There are also existing literatures on the CDMA-based underlay spectrum sharing approaches. The authors of [15] proposed a code assignment scheme for the SUs in the asynchronous CDMA underlay cognitive radio networks over multipath fading channels, which aims at minimizing interference caused to the PUs. The authors of [16] focused on the CDMA-based underlay cognitive radio systems where the PUs can increase transmit power to counterbalance the harmful interference caused by the SUs. In particular, they studied the relationship among the interference threshold of SUs, the transmit rate of SUs, and the transmit power of PUs. In [17], the authors proposed a two-phase channel and power allocation scheme for the underlay-based multi-cell cognitive radio networks to improve the system throughput which is defined as the total number of simultaneously served subscribers. In contrast to these existing works on the CDMAbased underlay spectrum sharing approaches, in this paper we formulate and tackle the more comprehensive joint power and rate allocation problems in the intrusive and non-intrusive spectrum sharing schemes for CDMA-based cognitive radio networks.

Channel sensing/probing, which provides the real-time spectrum information required by the spectrum sharing approaches, is also a critical part for the cognitive radio networks. In [10], we developed the efficient collaborative sensing scheme for the multi-channel wireless networks. The authors of [18] developed a game-theoretic sensing algorithm for multi-channel wireless networks. The authors of [19] proposed to use the spectrum sensor cluster to enhance the accuracy of the sensing outcome. The authors in [20] studied the optimal sensing duration which can maximize the SUs' achievable throughput. The authors of [21] presented an adaptive sensing scheduling scheme based on the channel conditions, which aims at maximizing the spectrum efficiency of the cognitive radio operations. The authors of [22] developed the relay sensing schemes that exploits the spatial diversity in different SUs to improve the spectrum sensing capabilities. The cooperative sensing strategies were also studied in [23], [24]. 


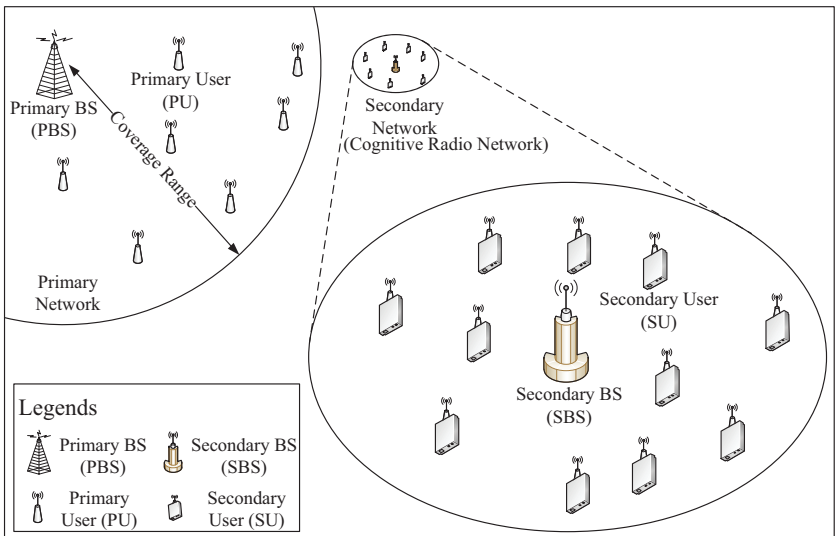

Fig. 1. Illustration of the CDMA-based secondary (users) network. The sub-figure on the bottom right corner shows the detailed topology of the secondary (users) network. The secondary (users) network consists of a set of SUs and a secondary base station (SBS). The SUs transmit data to the SBS by opportunistically accessing the licensed spectrum of the PUs in the primary (users) network.

Unlike the previous works, which utilize the channel sensing outcome only for the current transmission operation, in this paper we propose to utilize the channel sensing outcome not only to determine the spectrum sharing modes, but also to predict the PUs' activities (whether the PUs will re-occupy the spectrum during SUs' transmissions) to significantly reduce the interference caused by the SUs.

\section{THE SYSTEM MODELS}

\section{A. The Network Model}

We consider a single-hop cognitive radio network as shown in Fig. 1. The cognitive radio network consists of a set of SUs and a secondary base station (SBS). There is a primary network located adjacent to the cognitive radio network. In the primary network, a primary base station (PBS) broadcasts packets to its PUs through the downlink channel over a licensed spectrum band. In this paper, we mainly focus on the primary networks consisting of the stationary PBS and PUs, and thus assume that the secondary network has a priori knowledge about the locations of the stationary PBS and the stationary PUs, which is common and reasonable in many existing practical cases ${ }^{2}$, especially with the increasing support for cognitive radio networks by the governmental authority. In addition, we assume that the SBS has the dedicated downlink channel to communicate with the SUs without interfering the PUs. Thus, we do not need to consider the interference from the SBS to the PUs.

The PBS does not always occupy the channel. Due to the radio signal path loss and the PBS's frequent vacancy, the SUs may opportunistically utilize the licensed spectrum to access the SBS by using the intrusive/non-intrusive spectrum sharing modes. We assume that the distance between the PBS and

\footnotetext{
${ }^{2}$ As one of examples, the location of the stationary PBS and PUs in New York City can be found in http://www.city-data.com/city/New-YorkNew-York.html. For another example, in the fixed-WiMAX based last-hop broadband access networks, the locations for the stationary WiMAX basestation (i.e., PBS) and the stationary subscribed WiMAX end-users (i.e., PUs) can be known by inquiring the telecommunication carriers.
}

SBS is larger than the effective transmission range of the PBS. Under this assumption, the PBS has little interference on the uplinks of the cognitive radio network. The SUs communicate with the SBS through CDMA uplinks. As shown in Fig. 1, the PUs on the boundary, which is specified by the coverage range (see Fig. 1) of the primary network, between the primary network and the secondary network can be interfered by the SUs during the intrusive spectrum sharing mode operated by the cognitive radio network. We need to upper-bound the transmit power levels of the SUs to protect the right of the PUs on the boundary.

The downlink and uplink communications of the cognitive radio network is implemented in a time division manner. For the cognitive radio network, the time is partitioned into frames. Each frame includes an downlink subframe and a uplink subframe. During the downlink subframe, the SBS broadcasts the power allocations, and the decisions of operation modes to the SUs. Then, during the following uplink subframe, the SUs transmit packets with the assigned transmit power. The uplink communication is based on CDMA. Following the CDMA power allocations, the SBS can receive and correctly decode the SUs' packets simultaneously.

\section{B. The Primary Channel Model}

The licensed primary channel is modeled as an alternating renewal process which switches between $\mathrm{ON}$ and OFF states. The ON (or OFF) state represents that the PBS is (or is not) broadcasting packets to the PUs. The licensed channel state switches between ON and OFF states based on the PUs' activity. For an alternating renewal channel, let random variables $\tau_{1}$ and $\tau_{0}$ represent the sojourn times of $\mathrm{ON}$ and OFF states, respectively. Without loss of generality, we assume that $\tau_{1}$ is independent of $\tau_{0}$. Denote by $A_{\tau_{1}}(s)$ and $A_{\tau_{0}}(s)$ the probability density functions (pdf) for the durations of the licensed channel's ON state and OFF state, respectively. Then, we can get the probability, denoted by $u$, that the channel is in its ON state at an arbitrary time instance as follows:

$$
u=\frac{\int_{0}^{\infty} s A_{\tau_{1}}(s) d s}{\int_{0}^{\infty} s A_{\tau_{1}}(s) d s+\int_{0}^{\infty} s A_{\tau_{0}}(s) d s}=\frac{\bar{\tau}_{1}}{\overline{\tau_{1}}+\bar{\tau}_{0}},
$$

where $\bar{\tau}_{1}$ and $\bar{\tau}_{0}$ are the mean sojourn times of ON and OFF states, respectively. Note that in fact $u$ is the channel utilization with respect to (w.r.t.) PUs. The empirical spectrum measurement data [25], [26] already showed that the heavy-tailed distributions or exponential-tailed distributions are appropriate to characterize the duration of the licensed channel's ON/OFF state. In this paper, to better analyze our proposed scheme, we consider the gamma distribution and the two heavy-tailed distributions, namely, the pareto and lognormal distributions for the ON/OFF duration distribution of the primary networks.

\section{Channel Sensing and Data Transmission Models}

When the SUs perform the channel sensing, all of the SUs should keep their radios silent for a certain amount of time in order to obtain an accurate sensing outcome. During this silent period, no transmissions can be made. All of the SUs and SBS can participate in channel sensing so that the collaborative 
channel sensing can decrease the missed detection probability and false alarm probability.

Based on the selection of operation modes and sensing results, the SBS assigns power and rate to the active SUs that request to transmit packets the SBS. The SUs transmit data to the SBS simultaneously by using different CDMA codes according to the assigned power and rate. Denote by $\mathcal{N}$ the set of the active SUs that request for uplink communications. Denote by $W$ and $R_{i}$ the channel bandwidth and data rate for SU $i$, respectively. Thus, the processing gain for $\mathrm{SU} i$ is $W / R_{i}$. Let $P_{i}$ be the transmit power of SU $i$. Also, let $G_{j, 0}^{s s}$ be the channel gain between SU $j$ and the SBS. Thus, the signal to interference-plus-noise ratio (SINR), denoted by $\gamma_{i}^{s}$, of SU $i$ 's signal at the SBS can be obtained by [27]

$$
\gamma_{i}^{s}=\frac{W G_{i, 0}^{s s} P_{i}}{R_{i}\left(\frac{2}{3 R_{c}} \sum_{j \in \mathcal{N} \backslash\{i\}} G_{j, 0}^{s s} P_{j}+N_{0}\right)}
$$

where $R_{c}$ is the CDMA chip rate that is inversely proportional to the duration of a CDMA chip and $N_{0}$ is the power spectral density of a constant background noise.

\section{OUR Proposed Opportunistic SPeCtrum SHARING SCHEMES FOR UPLINK COGNITIVE MAC}

\section{A. The Overview of Our Proposed Opportunistic Spectrum Sharing Schemes}

Our proposed opportunistic spectrum sharing schemes, which aims at achieving the high spectrum utilization for cognitive radio networks, are the key components of the CDMA-based uplink cognitive MAC protocol. In our proposed scheme, the SUs can operate in two transmission modes: intrusive spectrum sharing mode and non-intrusive spectrum sharing mode. In the mode of non-intrusive spectrum sharing, the SUs can "share" the licensed spectrum with the PUs simultaneously by adapting the transmit power so that the interference caused by the SUs' transmissions is below the noise floor of the spectrum. The SBS makes the power and rate allocations with the constraint of PUs' interference constraints. In the intrusive spectrum sharing mode, the SUs sense the channel before transmitting. If the channel is idle, then the SBS can assign power to SUs without being confined by the PUs' interference constraints. We design rewarding functions to determine which spectrum sharing operation should be used by the SUs based on the channel utilization, the number of active SUs, and the interference constraints.

\section{B. Power and Rate Allocations}

We first focus on the power and rate allocations in CDMA uplink communications for the non-intrusive and the intrusive spectrum sharing schemes (modes) which are detailed in the following two cases, respectively.

\section{CASE 1: Intrusive Spectrum Sharing Mode}

Suppose that the power vector for the SUs are given. That is, the SINR of each SU before despreading at the SBS is fixed. We search for the optimized processing gain. For the convenience of presentation, rewriting Eq. (2), we have the
SINR, denoted by $\gamma_{i}^{s}$, of the received power of SU $i$ at the SBS as follows:

$$
\gamma_{i}^{s}=\frac{W G_{i, 0}^{s s} P_{i}}{R_{i}\left(\frac{2}{3 R_{c}} \sum_{j \in \mathcal{N} \backslash\{i\}} G_{j, 0}^{s s} P_{j}+N_{0}\right)}=\frac{s_{i} W}{R_{i}}
$$

where $s_{i}$ is the SINR of SU $i$ before despreading and is defined as

$$
s_{i} \triangleq \frac{G_{i, 0}^{s s} P_{i}}{\frac{2}{3 R_{c}} \sum_{j \neq i} G_{j, 0}^{s s} P_{j}+N_{0}} .
$$

Note that $\gamma_{i}^{s} \geq s_{i}$ because $\left(W / R_{i}\right) \geq 1$.

Let $\ell$ be the length of a packet. Let $\kappa_{i}$ be the instantaneous user-priority weighting factor for SU $i$. The larger the value of $\kappa_{i}$ for SU $i$ is, the higher priority SU $i$ has. We will elaborate on the determination of $\kappa_{i}$ in Section IV-G. The goodput, denoted by $\eta_{i}$, of SU $i$ can be written as:

$$
\eta_{i}=\kappa_{i} R_{i}\left[1-Y\left(\gamma_{i}^{s}\right)\right]^{\ell}=\kappa_{i} \frac{s_{i} W}{\gamma_{i}^{s}}\left[1-Y\left(\gamma_{i}^{s}\right)\right]^{\ell}
$$

where $Y(\cdot)$ is the bit error rate (BER) function, which is the function of SINR. The form of function $Y(\cdot)$ depends on the modulation and coding scheme used in the system. Our goal is to maximize the weighted goodput over all SUs. We formulate the optimization problem as follows:

$$
\begin{aligned}
\text { P0 : } & \max \\
& \sum_{i \in \mathcal{N}} \kappa_{i} \eta_{i} \\
\text { s.t. } & 0 \leq P_{i} \leq P_{i}^{\max }, \forall i \in \mathcal{N}
\end{aligned}
$$

where $\mathcal{N}$ is the set of the active SUs which request for uplink communications.

To solve the optimization problem P0, let us first concentrate on $\eta_{i}$ of a single SU $i$ in Eq. (5). On one hand, the first component, $\kappa_{i} s_{i} W / \gamma_{i}^{s}$, of the second equation in Eq. (5) is a decreasing function with $\gamma_{i}^{s}$. On the other hand, $Y\left(\gamma_{i}^{s}\right)$ is a decreasing function of $\gamma_{i}^{s}$. Thus, the second component, $\left[1-Y\left(\gamma_{i}^{s}\right)\right]^{\ell}$, of the second equation in Eq. (5) is an increasing function of $\gamma_{i}^{s}$. To find the value of $\gamma_{i}^{s}$ that achieves an extrema of $\eta_{i}$, we take the first-order derivative of $\eta_{i}$ given in Eq. (5) w.r.t. $\gamma_{i}^{s}$ :

$\frac{d \eta_{i}}{d \gamma_{i}^{s}}=\frac{-\kappa_{i} s_{i} W\left[1-Y\left(\gamma_{i}^{s}\right)\right]^{\ell-1}}{\left(\gamma_{i}^{s}\right)^{2}}\left[1-Y\left(\gamma_{i}^{s}\right)+\ell \gamma_{i}^{s} Y^{\prime}\left(\gamma_{i}^{s}\right)\right]$

where $Y^{\prime}(\cdot)$ is the derivative of the BER function $Y(\cdot)$. If setting Eq. (8) to be equal to 0 , then we have

$$
1-Y\left(\gamma_{i}^{s}\right)+\ell \gamma_{i}^{s} Y^{\prime}\left(\gamma_{i}^{s}\right)=0
$$

The roots of Eq. (9) are the extremas of $\eta_{i}$. The number of possible roots depends on the BER function $Y(\cdot)$ and $\ell$ only. Thus, the root for SU $i$ is also the root for any other SUs. Without loss of generality, let $\gamma_{i}^{s}=\gamma_{0}$, with $\gamma_{0}>0$, be one of the solutions, which achieves the local maximum of $\eta_{i}$. Since the processing gain should be less than or equal to 1 , we have $W s_{i} / \gamma_{0} \leq W$, resulting in $s_{i} \leq \gamma_{0}$. Thus, the optimal goodput for $\mathrm{SU} i$ is

$$
\eta_{i}= \begin{cases}\frac{\kappa_{i} s_{i} W}{\gamma_{0}}\left[1-Y\left(\gamma_{0}\right)\right]^{\ell}, & s_{i} \leq \gamma_{0} \\ \kappa_{i} W\left[1-Y\left(s_{i}\right)\right]^{\ell}, & s_{i}>\gamma_{0} .\end{cases}
$$


Based on Eq. (10), when $s_{i}>\gamma_{0}, \eta_{i}$ is not a linear function of $s_{i}$. We set $\left[1-Y\left(s_{i}\right)\right]^{\ell}$ to be a constant value. In this sense, $\eta_{i}$ is the linear function of $s_{i}$, implying that maximizing the objective function in $\mathbf{P 0}$ is equivalent to maximize $\sum_{i \in \mathcal{N}} \kappa_{i} s_{i}$ since we have the constant $W$ and $\gamma_{0}$ for each SU. Then, we have the following new power-allocation optimization problem.

Under the intrusive spectrum sharing mode, we can formulate the power-allocation optimization problem, denoted by P1, as follows:

$$
\begin{aligned}
\text { P1: } \max & \sum_{i \in \mathcal{N}} \kappa_{i} s_{i} \\
\text { s.t. } & 1) . \quad 0 \leq P_{i} \leq P_{i}^{\max }, \forall i \in \mathcal{N} \\
& 2) . \quad s_{i} \leq \gamma_{0}, \forall i \in \mathcal{N}
\end{aligned}
$$

By solving P1, we can derive the optimal SINR, denoted by $s_{i}^{\mathbf{P} 1}$, of SU $i$ under P1's mode. Then, substituting $s_{i}^{\mathbf{P} 1}$ into Eq. (10), we can obtain the optimal goodput, denoted by $\eta_{i}^{\mathbf{P 1}}$, for SU $i$ under P1's mode. It is worth noting that Eq. (11) is convex in each component $\left(P_{i}\right)$, but it is not convex over the entire power vector, which suggests that the Karush-KuhnTucker conditions are not sufficient for optimality. That is, taking partial derivatives cannot guarantee global optimality of a solution. Consequently, we have to optimize the nonconvex function in a very large space. Thus, we need to further analyze $\mathbf{P 1}$.

Theorem 1: The optimal solution to the objective function given by Eq. (11) lies on the surface of the polyhedron defined by the constraints given by Eqs. (12) and (13).

Proof: We derive the second-order derivative of the objective function with respect to $P_{i}$ as follows:

$$
\frac{\partial^{2}\left(\sum_{i \in \mathcal{N}} \kappa_{i} s_{i}\right)}{\partial P_{i}^{2}}=\sum_{j \in \mathcal{N} \backslash\{i\}} \frac{\kappa_{j} G_{j, 0}^{s s} P_{j}}{\left(\sum_{k \in \mathcal{N} \backslash\{j\}} G_{k, 0}^{s s} P_{k}+\frac{3 R_{c} N_{0}}{2}\right)^{3}} .
$$

It is clear that the second-order derivative of the objective function is always larger than 0 . Therefore, the objective function is convex w.r.t. $P_{i}$ and the optimal solution lies on the boundary, which completes the proof.

Theorem 1 characterizes the relationships among the given SU's transmit power, throughput, the interference that the SU imposes to the entire system, and the aggregate throughput over all SUs. By increasing a given SU's power, it increases its own data transmission rate while the data transmission rates of the other SUs decrease due to the additional multiple access interference (MAI) caused by the given SU. In contrast, the decrease of a given SU's transmit power can lead to the increase of data transmission rates of the other SUs because the MAI contributed by the given SU becomes smaller. Based on Theorem 1, when a given SU increases its transmit power, the objective function (i.e., weighted system data rate) maybe initially declines with the loss of data rate because the additional MAI impairs the increase of data rate caused by the new SU. However, when the SU keeps increasing transmit power, the benefit contributed by the SU eventually offsets the loss of data rate caused by the augmented MAI. Using Theorem 1, we can observe the following facts.
Fact 1: If $\max _{i \in \mathcal{N}}\left(\gamma_{i}^{s}\right)<\gamma_{0}$, then the optimal power allocation scheme for the intrusive spectrum sharing mode (i.e., P1) is as follows:

$$
P_{i} \in\left\{0, P_{i}^{\max }\right\}, \forall i \in \mathcal{N} .
$$

Fact 2: The objective function (Eq. (11)) of P1 is convex w.r.t. each component of the power vector. However, The objective function is not convex w.r.t. the entire power vector.

For the special case where $\kappa_{i}=\kappa_{j}, \forall i \neq j \in \mathcal{N}$, we can have a simple way to obtain the solution to P1. Without loss of generality, we suppose that

$$
\kappa_{i} G_{i, 0}^{s s} P_{i}^{\max }<\kappa_{j} G_{j, 0}^{s s} P_{j}^{\max }, \forall i<j
$$

Let $|\mathcal{N}|$ be the number of elements in $\mathcal{N}$. We can sort $|\mathcal{N}|$ active SUs based on the criteria given by Eq. (16) in the decreasing order of the weighted received power $\left(\kappa_{i} G_{i, 0}^{s s} P_{i}^{\max }\right)$. We call the first $n$ SUs in $\mathcal{N}$ as the preferred $n$ SUs, which are the best SUs in terms of weighted received power at the SBS. Denote by $\mathcal{N}_{\text {opt }}$, with $\mathcal{N}_{\text {opt }} \subset \mathcal{N}$, the set of SUs that transmit at their maximum power for maximizing objective function Eq. (11). There exists an $n_{\text {opt }}$, with $0<n_{\text {opt }}<$ $|\mathcal{N}|$, such that the optimal solutions can be achieved with $\mathcal{N}_{\text {opt }}=\left\{1,2, \cdots, n_{\text {opt }}\right\}$. That is, the optimal solution is achieved for the first $n_{\text {opt }}$ preferred SUs. Thus, for the case where $\kappa_{i}=\kappa_{j}, \forall i \neq j \in \mathcal{N}$, we can derive the optimal power allocation as follows: given the weights $\kappa_{i}$ and channel gain $G_{i, 0}^{s s}$, we sort the SUs based on Eq. (16). Then, we need to find the objective function achieved by the $n_{\text {opt }}$ preferred SUs. Letting $\mathcal{N}_{k}$ be the set of the first $k$ preferred SUs, we can derive $n_{\text {opt }}$ as follows:

$$
n_{\text {opt }}=\underset{1 \leq k \leq|\mathcal{N}|}{\arg \max } \sum_{1 \leq i \leq k} \frac{3 \kappa_{i} R_{c}}{2 \gamma_{0}} \frac{G_{i, 0}^{s s} P_{i}^{\max }}{\sum_{j \in \mathcal{N} \backslash\{i\}} G_{j, 0}^{s s} P_{j}^{\max }+3 N_{0} R_{c} / 2} .
$$

\section{CASE 2: Non-Intrusive Spectrum Sharing Mode}

Compared with the intrusive spectrum sharing operation mode, the non-intrusive spectrum sharing counterpart needs to take into consideration the power interference imposed to the PUs. We need to put more stringent power constraints to guarantee that the interference caused by the SUs is below the acceptable noise level in the view point of PUs. In other words, we need to add a constraint to guarantee that the SINR of the PUs in the interfering zone is higher than the pre-defined SINR threshold $\gamma_{t h}^{p}$.

Let $B$ be the transmit power of the PBS. Let $G_{j, i}^{s p}$ be the average channel gain between $\mathrm{SU} j$ and PU $i$. Let $G_{0, i}^{p p}$ be the average channel gain between the PBS and PU $i$. Since the information about the PBS and PUs is known to the secondary network, the average channel gain can be estimated based on the distance between the PBS and the PUs. Denote by $\mathcal{I}$ the set of PUs that will be interfered by SUs' intrusive transmissions. Due to the presence of the SUs and the corresponding MAI, we can write the SINR of PU $j$ as follows:

$$
\gamma_{i}^{p}=\frac{G_{0, i}^{p p} B}{\sum_{j \in \mathcal{N}} G_{j, i}^{s p} P_{j}+N_{0}}
$$

where $N_{0}$ is the power spectral density of a constant background noise. 


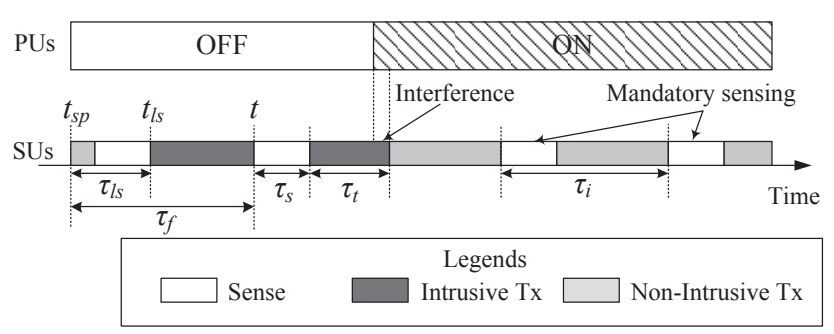

Fig. 2. Illustration of our proposed spectrum access schemes. The transmission duration $\tau_{t}$ depends on $\tau_{f}$ and the interference constraints. The SUs may interfere with the PUs when both the SUs and PUs access the spectrum.

Hence, we can formulate the power and rate optimization for the non-intrusive spectrum sharing operation as follows:

$$
\begin{aligned}
\text { P2: } \max & \sum_{i \in \mathcal{N}} \kappa_{i} s_{i} \\
\text { s.t. } & 1) .0 \leq P_{i} \leq P_{i}^{\max }, \forall 1 \in \mathcal{N} \\
& \text { 2). } \gamma_{i}^{s} \geq \gamma_{0}, \forall i \in\left\{k: P_{k}>0\right\} \\
& \text { 3). } \gamma_{i}^{p} \geq \gamma_{t h}^{p}, \forall i \in \mathcal{I}
\end{aligned}
$$

Similar to $\mathbf{P 1}$, the objective function of $\mathbf{P 2}$ is also not convex in the power vector. The solution to $\mathbf{P 2}$ also lies on the boundary determined by the constraints. We can use a feasible-directions-based method [28] to solve the non-convex optimization problem of $\mathbf{P 2}$. Solving $\mathbf{P 2}$, we can derive the optimal SINR, denoted by $s_{i}^{\mathbf{P 2}}$, of SU $i$ under P2's mode. Substituting $s_{i}^{\mathbf{P 2}}$ to Eq. (10), we can get the optimal goodput, denoted by $\eta_{i}^{\mathbf{P 2}}$, for SU $i$ under P2's mode. Note that when the distances between the SU transmitters and the PU receivers are far enough, the power and rate optimization problem $\mathbf{P 2}$ for the non-intrusive spectrum sharing mode reduces to the power and rate optimization problem $\mathbf{P 1}$ for the intrusive spectrum sharing mode.

\section{The Interference Probability of Intrusive Spectrum Sharing Operations}

Note that in the intrusive spectrum sharing operation, the SUs may interfere with the PUs because the SUs and PUs are not synchronized. We need to investigate the interfering probability of the intrusive spectrum sharing operations. Let $t_{s p}$ be the most recent time when the channel switches from ON to OFF state and $t_{l s}$ be the most recent time that the channel is sensed. Fig. 2 shows the relationship among the important notations and variables in the spectrum sharing schemes. If the channel is in OFF state at $t_{l s}$, then the probability that the channel remains OFF for a certain amount of time is non-zero, and thus it is possible for the SUs to perform intrusive spectrum operation. Let $\tau_{s}$ and $\tau_{t}$ be the durations needed by the SUs to sense the channel and transmit data packets, respectively. Given that the SUs start sensing the channel at time $t$, during the channel sensing period, we can derive the probability, denoted by $I(t)$, that the channel keeps on being in OFF state as follows:

$$
\begin{aligned}
I(t) & =\frac{\operatorname{Pr}\left\{\text { duration of OFF state }>t+\tau_{s}-t_{s p}\right\}}{\operatorname{Pr}\left\{\text { duration of OFF state }>t_{l s}-t_{s p}\right\}} \\
& =\frac{\int_{t+\tau_{s}-t_{s p}}^{\infty} A_{\tau_{0}}(s) d s}{\int_{t_{s}-t_{s p}}^{\infty} A_{\tau_{0}}(s) d s}=\frac{1-C_{\tau_{0}}\left(t+\tau_{s}-t_{s p}\right)}{1-C_{\tau_{0}}\left(t_{l s}-t_{s p}\right)},
\end{aligned}
$$

where $C_{\tau_{0}}(s)$ is the cumulative distribution function (cdf) of the duration of the channel's OFF state.

During the entire duration of $\tau_{s}$, if the channel is in OFF state, the SUs consider that the channel is idle and can be opportunistically utilized. In other words, if the channel remains idle after the channel sensing, based on our proposed spectrum sharing schemes, the SUs can perform the intrusive spectrum sharing operations. The SUs may interfere with the PUs if the PBS resumes broadcasting packets to PUs again (i.e., the channel transits from OFF to ON) during the SUs' transmissions. Denote by $Q\left(\tau_{t}, t\right)$ the probability that the SUs which send packets between $t$ and $t+\tau_{t}$ will interfere with PUs, given that the channel is sensed idle at the beginning of SUs' attempt to access the channel. $Q\left(\tau_{t}, t\right)$ is equal to the probability that the channel state flips from OFF to ON during SUs' opportunistic transmission, which can be derived as:

$$
\begin{aligned}
Q\left(\tau_{t}, t\right) & =1-\frac{\operatorname{Pr}\left\{\text { duration of OFF state }>\left(t+\tau_{t}-t_{s p}\right)\right\}}{\operatorname{Pr}\left\{\text { duration of OFF state }>t-t_{s p}\right\}} \\
& =\frac{C_{\tau_{0}}\left(t+\tau_{t}-t_{s p}\right)-C_{\tau_{0}}\left(t-t_{s p}\right)}{1-C_{\tau_{0}}\left(t-t_{s p}\right)}
\end{aligned}
$$

and has the following property as described in Proposition 1.

Proposition 1: $Q\left(\tau_{t}, t\right)$ is a monotonically increasing function of $\tau_{t}$.

Proof: Taking a derivation of $Q\left(\tau_{t}, t\right)$ w.r.t. $\tau_{t}$, we get

$$
\frac{\partial Q\left(\tau_{t}, t\right)}{\partial \tau_{t}}=\frac{A_{\tau_{0}}\left(t+\tau_{t}-t_{s p}\right)}{1-C_{\tau_{0}}\left(t-t_{s p}\right)} .
$$

Since $A_{\tau_{0}}(x)>0$ and $0 \leq C_{\tau_{0}}(x)<1, \forall x, Q\left(\tau_{t}, t\right)$ is a monotonically increasing function of $\tau_{t}$.

Proposition 1 states that as the transmission duration $\tau_{t}$ of the intrusive spectrum sharing mode increases, the probability that the SUs interfere with SUs increases.

\section{The Optimal Transmission Duration of the Intrusive Spec- trum Sharing Mode}

Since the SUs are not synchronized with the PUs, the probability $Q\left(\tau_{t}, t\right)$ that the SUs interfere with PUs is non-zero, and thus the SUs may inevitably cause harmful interference imposed onto PUs in the intrusive spectrum sharing mode. We have to limit the maximum interference that is caused by SUs. We consider two interference constraints, such as power constraint and time constraint. In the non-intrusive spectrum sharing, we limit the transmit power of SUs so that the interference temperature is below PU's tolerable noise power, as specified in $\mathbf{P 2}$. For the intrusive spectrum sharing, we apply the constraint in time domain by limiting the amount of time when the SUs and PUs transmit simultaneously.

We define $\tau_{f} \triangleq t-t_{s p}$, which is illustrated in Fig. 2, as the duration elapsed from the most recent channel switchingtime point to the time when SUs attempt to access the channel. Given that the channel is idle at the beginning of SUs' attempt 
to access channel and the channel-switch occurs during the transmission, we can obtain the pdf, denoted by $L_{\tau_{t}, \tau_{f}}(s)$, for the residual time of the PUs' OFF state given that the channelswitch occurs during the SUs' transmission as follows:

$$
L_{\tau_{t}, \tau_{f}}(s)= \begin{cases}\frac{A_{\tau_{0}}\left(s+\tau_{f}\right)}{C_{\tau_{0}}\left(\tau_{f}+\tau_{t}\right)-C_{\tau_{0}}\left(\tau_{f}\right)}, & 0<s \leq \tau_{t} \\ 0, & \text { otherwise }\end{cases}
$$

Then, we can derive the average duration, denoted by $D\left(\tau_{t}, \tau_{f}\right)$, that the SUs interfere with the PUs as Eq. (27), where $Q\left(\tau_{t}, t_{s p}+\tau_{f}\right)$ is the probability that PUs are interfered by SUs' transmission in the intrusive spectrum sharing mode, and is given by Eq. (24). For different distributions, we derive a set of closed-form expressions for Eq. (27) in Appendix A.

As time elapses, the probability that the SUs interfere with PUs changes. The transmission time $\tau_{t}$ of the SUs should be dynamically adjusted with the change of probability that the SUs interfere with PUs. Let $D_{t h}$ be the interference parameter for the intrusive spectrum sharing mode. Based on Eq. (27), let $\tau_{t}^{*}$ be the maximum allowable transmission time of the intrusive spectrum sharing mode given $\tau_{f}$, i.e.,

$$
\tau_{t}^{*}=\min \left\{\tau_{t}^{\max }, \max \left\{\tau_{t}: D\left(\tau_{t}, \tau_{f}\right) \leq D_{t h}\right\}\right\}
$$

where $\tau_{t}^{\max }$ is the pre-defined maximum transmission time. It is worth noting that the larger $D_{t h}$ suggests a longer transmission duration that the SUs can use, but also the more interference caused to the PUs.

In our proposed scheme, both the intrusive and non-intrusive spectrum sharing modes use the CDMA-based access technology and use the same spectrum bandwidth that is licensed to the primary network. These two sharing modes share most of the transmission and PHY designs. The major difference between the intrusive and non-intrusive spectrum sharing modes in terms of transmission parameters of the cognitive radio is the transmit power levels. Since the transmit power can be easily adjusted in cognitive radio, it is feasible to switch between the intrusive and non-intrusive sharing modes in a real-time manner. In particular, the transmit power adjustment of a cognitive radio can be implemented in a significantly shorter time period as compared with the operation periods of the intrusive and non-intrusive spectrum sharing modes. Thus, the power-switch time between the intrusive spectrum sharing mode (P1) and the non-intrusive spectrum sharing mode (P2) has virtually no impact on the performance of our proposed scheme.

\section{E. The Selection Between the Intrusive Spectrum Sharing Mode and the Non-Intrusive Spectrum Sharing Mode}

Under our proposed dynamic spectrum access schemes, the SUs can select between the non-intrusive spectrum access mode and the intrusive spectrum mode. This section describes how the SUs select the operation modes. We proceed the descriptions in the following two cases, respectively.

\section{Case (1): The primary channel is in ON state}

Under this case where the primary channel is in ON state, the only available operation mode for the SUs is non-intrusive spectrum sharing mode. If the primary channel is sensed as ON state, the SUs use the non-intrusive spectrum sharing mode where the total transmit power of the SUs is below the noise floor for the PUs.

\section{Case (2): The primary channel is in OFF state}

In the case where the primary channel is in OFF state, the SUs can have two options in accessing the primary channel: intrusive or non-intrusive spectrum sharing modes. The selection principle is that the SUs choose the spectrum sharing mode which leads to higher goodput to transmit data packets to the SBS.

Because the intrusive spectrum sharing mode always starts with channel sensing and the sensing duration is constant, as the transmission time decreases, the communication overhead increases. When the transmission time keeps on decreasing, the goodput of the intrusive spectrum sharing mode will eventually be equal to that of the non-intrusive spectrum sharing mode, and then become lower than that of the nonintrusive spectrum sharing mode. Note that the non-intrusive spectrum sharing mode does not require to sense the channel before transmissions. Then, we can construct a rewarding function, denoted by $\zeta(t)$, in terms of goodput of the nonintrusive spectrum sharing mode as follows:

$$
\zeta(t)=\sum_{i \in \mathcal{N}} \eta_{i}^{\mathbf{P 2}}
$$

where $\eta_{i}^{\mathbf{P 2}}$ is the optimal goodput of SU $i$ under $\mathbf{P 2}$, which can be obtained by solving the optimization problem $\mathbf{P 2}$ given in Section IV-B. We can construct another rewarding function, denoted by $\theta(t)$, in terms of the goodput of the intrusive spectrum sharing mode as follows:

$$
\theta(t)=I(t)\left(\frac{\tau_{t}^{*}}{\tau_{s}+\tau_{t}^{*}}\right) \sum_{i \in \mathcal{N}} \eta_{i}^{\mathbf{P 1}}+[1-I(t)]\left(\frac{\tau_{u}-\tau_{s}}{\tau_{u}}\right) \zeta(t) .
$$

where $\eta_{i}^{\mathbf{P 1}}$ is the optimal goodput of SU $i$ under $\mathbf{P 1}$, which can be obtained by solving the optimization problem $\mathbf{P 1}$ given in Section IV-B, and $\tau_{u}$ is the maximum transmission duration in the non-intrusive spectrum sharing mode. As shown in Eq. (30), the goodput of the intrusive spectrum sharing mode is the sum of the following two terms. The first term on the right hand side of Eq. (30) represents that the SUs can use the higher transmit power to access the channel if the channel keeps idle after the channel sensing. In particular, $\left[\tau_{t}^{*} /\left(\tau_{s}+\tau_{t}^{*}\right)\right]$ in the first term on the right hand side of Eq. (30) suggests the

$$
\begin{aligned}
D\left(\tau_{t}, \tau_{f}\right) & =\int_{0}^{\tau_{t}} Q\left(\tau_{t}, t_{s p}+\tau_{f}\right) L_{\tau_{t}, \tau_{f}}(s)\left(\tau_{t}-s\right) d s=Q\left(\tau_{t}, t_{s p}+\tau_{f}\right)\left[\left(\tau_{t}+\tau_{f}\right)-\frac{1}{C_{\tau_{0}}\left(\tau_{f}+\tau_{t}\right)-C_{\tau_{0}}\left(\tau_{f}\right)} \int_{\tau_{f}}^{\tau_{t}+\tau_{f}} t A_{\tau_{0}}(t) d t\right] \\
& =\frac{1}{1-C_{\tau_{0}}\left(\tau_{f}\right)}\left\{\left(\tau_{t}+\tau_{f}\right)\left[C_{\tau_{0}}\left(\tau_{f}+\tau_{t}\right)-C_{\tau_{0}}\left(\tau_{f}\right)\right]-\int_{\tau_{f}}^{\tau_{t}+\tau_{f}} t A_{\tau_{0}}(t) d t\right\}
\end{aligned}
$$


overhead of the channel sensing. On the other hand, the second term on the right hand side of Eq. (30) indicates that the SUs have to use lower transmit power to share the spectrum with PUs if the channel is sensed as ON state.

Based on the outcome of $\zeta(t)$ and $\theta(t)$ at time $t$, the SBS selects the spectrum sharing mode which yields the higher goodput. If $\tau_{t}^{*}$ is a decreasing function of $\tau_{f}$, then the SBS will always choose the non-intrusive spectrum sharing mode over the intrusive spectrum sharing mode when $\tau_{f}$ is larger than a certain threshold. In other words, the SUs will more likely choose the non-intrusive spectrum sharing mode without channel sensing.

\section{F. Mandatory Channel Sensing}

Since the information about the channel switching-time point is critical to our proposed schemes, it is mandatory for the SUs to periodically sense the channel to detect/estimate the channel switching-time point in both cases where the primary channel is in ON or OFF states, which are detailed in the following two cases, respectively.

\section{Case I: The primary channel is in ON state}

Although the non-intrusive spectrum sharing mode does not require channel sensing, the periodical channel sensing is still necessary in the case where the primary channel is in ON state, because the SUs are required to have the knowledge of the time when the channel switches from ON to OFF state. The SUs must frequently sense the channel. Fig. 2 illustrates an example of the mandatory sensing when the primary channel is in ON state. The sensing period, denoted by $\tau_{i}$, depends on the time elapsing from the last time when the channel switches from OFF to ON state.

Denote by $t_{s p}^{\prime}$ and $t_{s p}$ the old channel switching-time point and the new estimated channel switching-time point between the two consecutive channel sensings, respectively. We can derive the probability, denoted by $P_{c s}$, that the channel-switch occurs between two consecutive channel sensings as follows:

$$
P_{c s}=\frac{C_{\tau_{1}}\left(\tau_{l s}+\tau_{i}\right)-C_{\tau_{1}}\left(\tau_{l s}\right)}{1-C_{\tau_{1}}\left(\tau_{l s}\right)}
$$

where $\tau_{l s} \triangleq t_{l s}-t_{s p}^{\prime}$ is the duration between the most recent sensing time and the most recent channel switching time, and $C_{\tau_{1}}(t)$ is the cdf of the licensed channel's ON state. If the channel-switch occurs between two consecutive channel sensings and $\tau_{l s}$ is given, we can derive the pdf, denoted by $p_{t_{s p}}\left(s, \tau_{l s}\right)$, of the new channel switching-time point as follows:

$$
p_{t_{s p}}\left(s, \tau_{l s}\right)= \begin{cases}\frac{A_{\tau_{1}}\left(s+\tau_{l s}\right)}{C_{\tau_{1}}\left(\tau_{l s}+\tau_{i}\right)-C_{\tau_{1}}\left(\tau_{l s}\right)}, & 0<s \leq \tau_{i} \\ 0, & \text { otherwise }\end{cases}
$$

The channel sensing frequency (or channel sensing period) determines the accuracy of the estimated channel switchingtime point. From the view point of non-intrusive spectrum sharing, the channel sensing is not helpful and is thus considered as the pure overhead, which eventually results in degradation of the goodput. There is a tradeoff between the accuracy of the estimated channel switching-time point and the goodput. Specifically, if we have the higher channel sensing frequency, then we can have the more accurate channel switching-time point, but lower goodput. In contrast, the lower channel sensing frequency leads to the more estimation error of the channel switching-time point and the higher goodput. Intuitively, when $\tau_{l s}$ is small, the probability that the channel switches from OFF to ON state is small. Thus, a low channel sensing frequency is good enough to estimate the channel switching-time point. On the other hand, when $\tau_{l s}$ is high, the probability that the channel switches from OFF to ON state is high, which implies that the high channel sensing frequency is necessary to accurately detect the channel switching-time point. In order to minimize the goodput degradation caused by the channel sensing while guaranteeing the accuracy of the estimated channel switching-time point, we introduce the mean squared estimation error for the channel switching-time point to qualitatively derive the channel sensing period $\tau_{i}$ based on the elapsed time $\tau_{l s}$ away from the last channel switching-time point. We derive the mean squared estimation error, denoted by $\epsilon\left(\tau_{i}, t_{s p}\right)$, of $t_{s p}$ as follows:

$$
\begin{aligned}
\epsilon\left(\tau_{i}, t_{s p}\right)= & \int_{0}^{\tau_{i}}\left(t_{s p}-t_{l s}-s\right)^{2} p_{t_{s p}}\left(s, \tau_{l s}\right) d s \\
= & \frac{2\left(t_{s p}-t_{s p}^{\prime}\right) \int_{\tau_{l s}}^{\tau_{l s}+\tau_{i}} t A_{\tau_{1}}(t) d t-\int_{\tau_{l s}}^{\tau_{l s}+\tau_{i}} t^{2} A_{\tau_{1}}(t) d t}{C_{\tau_{1}}\left(\tau_{l s}\right)-C_{\tau_{1}}\left(\tau_{l s}+\tau_{i}\right)} \\
& \quad+\left(t_{s p}-t_{s p}^{\prime}\right)^{2}
\end{aligned}
$$

where $t_{l s} \leq t_{s p} \leq t_{l s}+\tau_{i}$, and $p_{t_{s p}}(s)$ is the pdf of $t_{s p}$ given by Eq. (32). For different distributions, we derive a set of the closed-form expressions for Eq. (33) in Appendix A.

Let $t_{s p}^{*}\left(\tau_{i}\right)$ be the optimal $t_{s p}$ that minimizes $\epsilon\left(\tau_{i}, t_{s p}\right)$ for the given $\tau_{i}$, which implies:

$$
t_{s p}^{*}\left(\tau_{i}\right)=\underset{0 \leq t_{s p} \leq \tau_{i}}{\arg \min } \epsilon\left(\tau_{i}, t_{s p}\right)
$$

Define $\epsilon_{t h}$ as the pre-defined threshold for the mean squared error of estimated channel switching time. Thus, when the non-intrusive spectrum sharing mode is adopted, the optimal sensing period is

$$
\tau_{i}^{*}=\max \left\{\tau_{i}: \epsilon\left(\tau_{i}, t_{s p}^{*}\left(\tau_{i}\right)\right) \leq \epsilon_{t h}\right\}
$$

\section{Case II: The primary channel is in OFF state}

When the primary channel is in OFF state, we can use the intrusive spectrum sharing mode which requires the channel sensing before SUs' transmissions. In this case, the channel sensing is part of the channel accessing process. Essentially, the channel sensing frequency required by the intrusive spectrum sharing mode is higher than that required for channel switching-time point detection/estimation. In this sense, the mandatory channel sensing can be already covered by the channel sensing required in intrusive spectrum sharing mode.

\section{G. The Fairness}

Our proposed scheme takes into account the fairness. Since different SUs may have different service requirements, we employ a set of weighting factors $\omega_{i}$ to represent the different priorities of the SUs. Our proposed system aims at achieving the long-term fairness by updating the instantaneous userpriority weighting factor $\kappa_{i}$.

Definition 1: The long-term fairness is said to be achieved if during a long duration of time, the ratios of the aggregated 
transmission rate to the weighting factor for all SUs are the same, i.e.,

$$
\lim _{n \rightarrow \infty} \frac{\sum_{n} R_{i}(n)}{\omega_{i}}=\lim _{n \rightarrow \infty} \frac{\sum_{n} R_{j}(n)}{\omega_{j}}, \forall i \neq j \in \mathcal{S}
$$

where $R_{i}(n)$ is data rate of the $\mathrm{SU} i$ at the $n$-th round of spectrum-access and $\mathcal{S}$ is the index set of all SUs.

To satisfy Eq. (36), we need to dynamically adjust the instantaneous weights that are used in the optimization problems including $\mathbf{P 1}$ and $\mathbf{P 2}$. Let $S_{i}(n)$ be the average transmission rate for SU $i$ during the pervious $n$ times of attempting transmissions. The SBS updates $S_{i}(n)$ for SU $i$ once the power allocation decision is made provided that $\mathrm{SU} i$ has a request for transmission. Note that the SBS does not update $S_{i}(n)$ for SU $i$ at the round in which SU $i$ does not have anything to send to SBS. We adopt the Exponential Moving Average (EMA) to update $S_{i}(n)$ as follows:

$$
S_{i}(n)=\delta R_{i}(n)+(1-\delta) S_{i}(n-1)
$$

where $\delta$, with $0 \leq \delta \leq 1$, is a pre-defined constant smoothing factor. Under EMA, the weighting for the older transmission rate decreases exponentially, which implies that the unfairness caused by recent power allocations is more important than the unfairness contributed by older power allocations. In this sense, the fairness scheme will put more efforts to compensate for the unfairness caused by recent power allocations. Thus, the instantaneous user-priority weighting factor $\kappa_{i}$ for SU $i$ at the $(n+1)$-th spectrum access attempt can be updated by setting:

$$
\kappa_{i}=\frac{\omega_{i}}{S_{i}(n)}
$$

\section{H. The Details of Our Proposed Scheme}

Figure 3 shows the detailed pseudo-code of our proposed scheme. There are two important types of events occurring in our system: 1) the event of sensing and 2) the event of spectrum sharing mode selection. The objective of our proposed scheme is to handle these two types of events by repeatedly updating the parameters for the events. Let $t_{s}$ and $t_{m}$ be the triggers for the events of sensing and spectrum sharing mode selection, respectively. Initially, we set both of the last sensing time $t_{l s}$ and last channel switching-time point $t_{s p}$ to negative infinity, the sensing trigger $t_{s}$ to zero, and spectrum sharing mode selection trigger $t_{m}$ to positive infinity.

If the current time is equal to $t_{s}$, then the sensing event is triggered. The SUs sense the channel for a duration of $\tau_{s}$. If the channel switches states during the sensing, the SUs update $t_{s p}$ to the channel switching-time point which the SUs have just detected. Otherwise, if the channel sensing result is different from the last sensing result, then the system calculates the new estimated $t_{s p}$ based on Eq. (34). Then, based on the channel state, the systems choose the transmit power. If the channel is idle, the SUs transmit packets to the SBS by using the transmit power according to the optimal solution to $\mathbf{P 1}$ with a duration of $\tau_{t}^{*}$ which is calculated by using Eq. (28). In contrast, if the channel is busy, the SUs use a low transmit power given by the optimal solution to $\mathbf{P 2}$ to access the channel. The SUs
00. Initialize: $t_{l s}=-\infty, t_{s p}=-\infty, t_{s}=0, t_{m}=\infty$

01. while(1)

02. $\quad t:=$ current time

03. if $t=t_{s} / /$ triggered by sensing event

04. sense the channel for the duration of $\tau_{s}$

05. if channel switches states during sensing update $t_{s p}$ to be the new channel switching-time point

if channel state is different from the last sensing result

calculate $t_{s p}$ based on Eq. (34)

update $t:=t+\tau_{s}, t_{l s}:=t$

calculate $\tau_{i}$ based on Eq. (35)

if channel is idle

update $\tau_{f}:=t-t_{s p}$

calculate $\tau_{t}^{*}$

update $t_{m}:=t+\tau_{t}^{*}, t_{s}:=t+\tau_{i}$

assign power and rate according to $\eta_{i}^{\mathbf{P 1}}$ else

SUs send packets to SBS using intrusive mode for $\tau_{t}^{*}$

update $t_{s}:=t+\tau_{i}$

assign power and rate according to $\eta_{i}^{\mathbf{P} 2}$

SUs send packets to SBS using non-intrusive mode

if $t=t_{m} / /$ triggered by selection of spectrum sharing mode update $\tau_{f}:=t-t_{s p}$

calculate $\eta_{i}^{\mathbf{P 1}}$ and $\eta_{i}^{\mathbf{P 2}}, \forall i \in \mathcal{N}$

calculate $\theta(t), \zeta(t)$

if $\theta(t)<\zeta(t)$

assign power and rate according to $\eta_{i}^{\mathbf{P 2}}$

SUs send packets to SBS using non-intrusive mode

update $t_{m}:=\infty$

else

$/ /$ keep using non-intrusive until next mandatary sensing

update $t_{s}:=t / /$ sense the channel immediately

Fig. 3. The pseudo code of our proposed schemes for the selection between the intrusive spectrum sharing mode and the non-intrusive spectrum sharing mode.

also need to update the new sensing trigger $t_{s}$ and selection trigger $t_{m}$.

The event of selecting the spectrum sharing mode is triggered if the current time is equal to $t_{m}$. There are two options for the SUs: 1) trying to use the intrusive spectrum sharing mode by sensing the channel first, or 2) immediately sending packets to the SBS with a lower transmit power. The SBS calculates and compares $\zeta(t)$ with $\theta(t)$. On one hand, if $\theta(t)$ is larger than $\zeta(t)$, then the SUs use the non-intrusive spectrum sharing mode to access the channel. Furthermore, $t_{m}$ is set to be infinity, which suggests that before the next mandatory channel sensing, the SUs keep on using the non-intrusive spectrum sharing mode. On the other hand, if $\zeta(t)$ is larger than $\theta(t)$, then the SUs will try to use the intrusive spectrum sharing mode by setting the sensing trigger $t_{s}$ to be the current time.

\section{Performance Evaluations}

To conduct the performance evaluations, we simulate our proposed scheme by using the OMNeT++ [29] simulator. Our simulated primary and secondary networks have the similar wireless networks topologies as shown in Fig. 1. In particular, there is one primary channel with the bandwidth of $50 \mathrm{MHz}$ licensed to the primary network. There are 1 PBS and 3 PUs in the primary network and there are 1 SBS and 10 SUs in the secondary network. The SUs form the wireless-network cell topology with the SBS being at the center position of the wireless-network cell. The average distance between the SUs and the SBS is 1 unit of distance. The average distance 


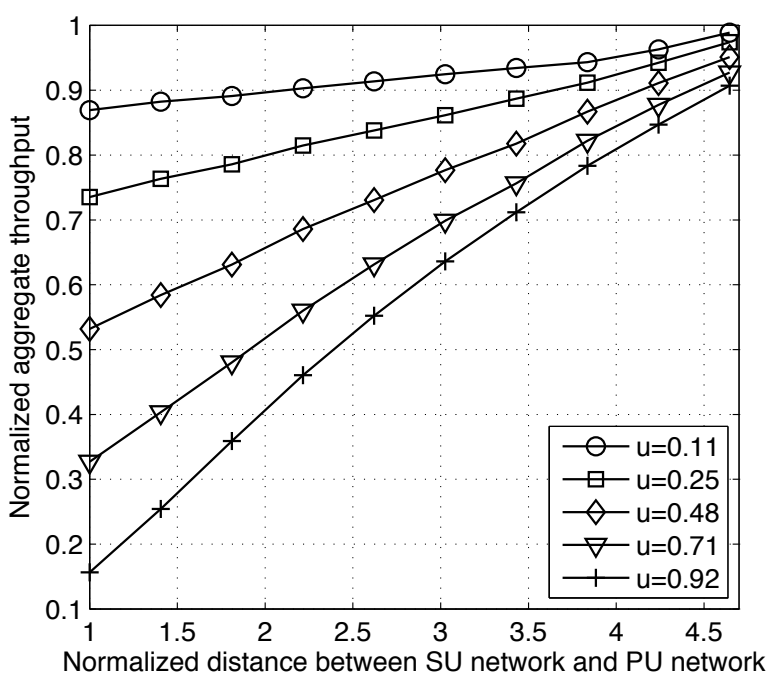

Fig. 4. The impact of distance between the secondary network and primary network on the normalized aggregate throughput of our proposed scheme when the PUs' traffic follows lognormal distribution.

between PUs and the SBS is 4.3 units of distance. In the simulations, we set $G_{i, 0}^{s s}=G_{j, 0}^{s s}$ and $P_{i}^{\max }=P_{j}^{\max }, \forall i \neq j$. The sensing duration is $0.5 \mathrm{~ms}$. The priority weighting factor $\omega_{i}$ for each SU is set to be $\omega_{i}=1, \forall i \in \mathcal{S}$ (i.e., the equalshare fairness). Each $\mathrm{SU}$ is the persistent data source which always has data to send with the non-empty queue, implying that each SU always requests to transmit data to the SBS.

We first study the impact of the distance between the primary network and secondary network on the throughput of our proposed scheme. Fig. 4 plots the normalized aggregate throughput of our proposed scheme against the normalized distance between the secondary network and primary network with different channel utilizations of PUs $(u)$ when the PUs' traffic follows lognormal distribution. Note that the aggregate throughputs in this paper are normalized by the throughput of the complete intrusive spectrum accessing mode with zero channel utilization of PUs. In addition, the distances between the SU and primary networks are normalized by the average distance between the SBS and SUs. As shown in Fig. 4, the normalized aggregate throughput increases as the normalized distance between the secondary network and primary network increases because the larger distance between the SU and primary networks leads to the larger throughput of the nonintrusive spectrum sharing mode. Furthermore, from Fig. 4, as the distance between the SU and primary networks increases, the difference of throughputs between the higher channel utilization of PUs and lower channel utilization of PUs decreases, which is because of the following reasons. When the distance between the secondary network and primary network is small, the difference among the throughputs achieved by the intrusive and non-intrusive spectrum sharing modes is large, implying that with lower channel utilization of PUs, the SUs spending more time in non-intrusive spectrum sharing mode achieves much less throughput than the SUs with higher channel utilization of PUs. In contrast, when the distance between the secondary network and primary network is large,

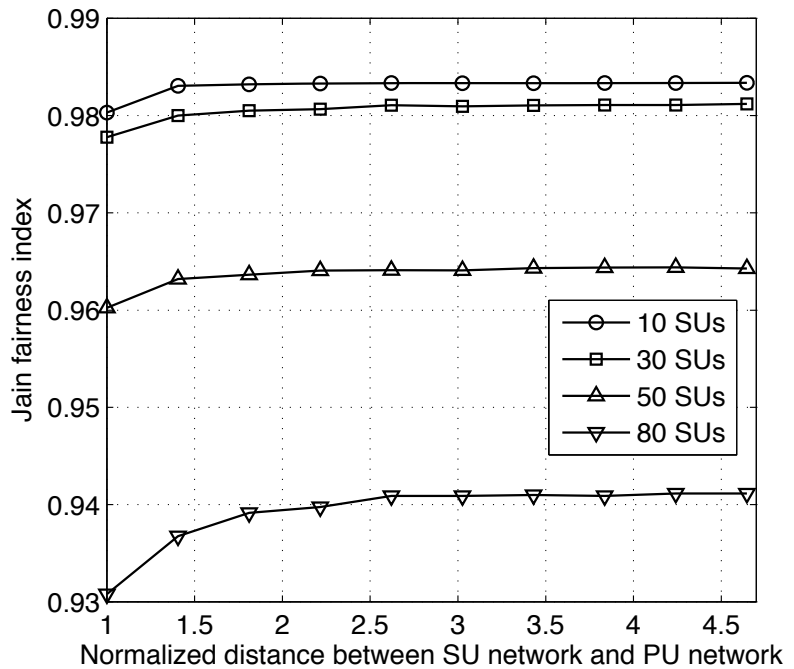

Fig. 5. The Jain fairness index vs. the distance between the secondary network and primary network. The channel utilization of PUs is 0.17 and the PUs' traffic follows lognormal distribution.

the throughput of the intrusive spectrum mode is close to the throughput of the non-intrusive spectrum sharing mode. As a result, the channel utilization of PUs has less impact on the throughput when the distance between the secondary network and primary network is larger.

Then, we evaluate the fairness performance of our proposed scheme using the Jain fairness index [30] (i.e., the equal-share attains the maximum Jain fairness index). Fig. 5 plots the Jain fairness index against the distance between the secondary network and primary network with the different numbers of SUs in the secondary network. The channel utilization of PUs is 0.17 and the PUs' traffic follows lognormal distribution. As shown in Fig. 5, our proposed scheme can achieve the desired farness among SUs in the secondary network. In Fig. 5, for a given number of SUs, the Jain fairness index slightly increases as the distance between the secondary and primary networks increases, which is due to the following reasons. When the distance between the secondary network and primary network gets larger, the interference-level constraint on the secondary network becomes more relaxed. This implies that the difference between the SU-achieved throughputs under the non-intrusive and intrusive spectrum sharing modes for each round of spectrum-access becomes smaller. Therefore, when the distance between the secondary network and primary network gets larger, regardless of the higher or lower probabilities for SUs to use the intrusive or non-intrusive spectrum sharing modes, the statistically-averaged throughputs for all SUs intend to converge to the equal-share fairness. Thus, the larger the distance between the secondary and primary networks, the larger the Jain fairness index. Fig. 5 also shows that the more the SUs in the secondary network, the lower the Jain fairness index. It is expected because of the following reasons. With limited wireless resources, when the number of SUs becomes larger, the probability for each SU to actually use the spectrum decreases for each round of spectrum-access opportunity, resulting in the lower Jain fairness index. 


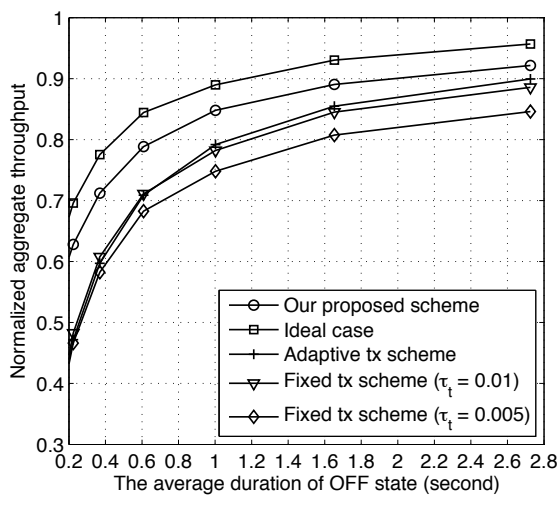

(a)

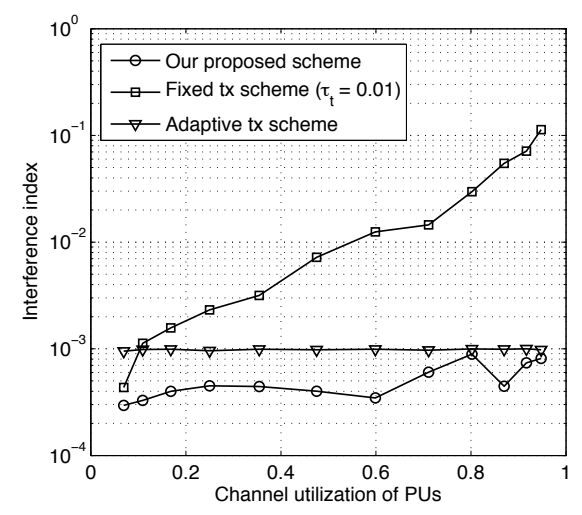

(d)

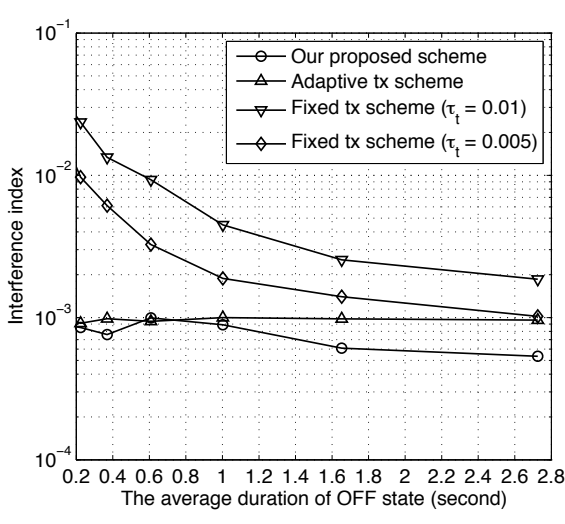

(b)

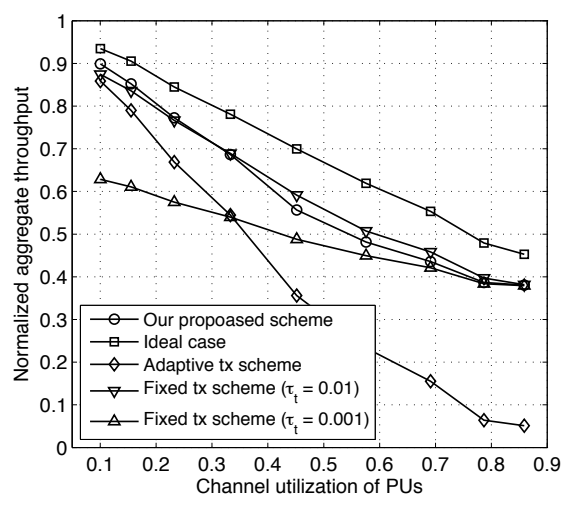

(e)

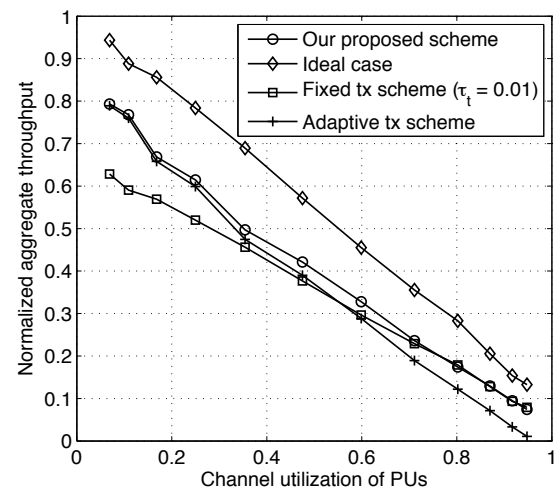

(c)

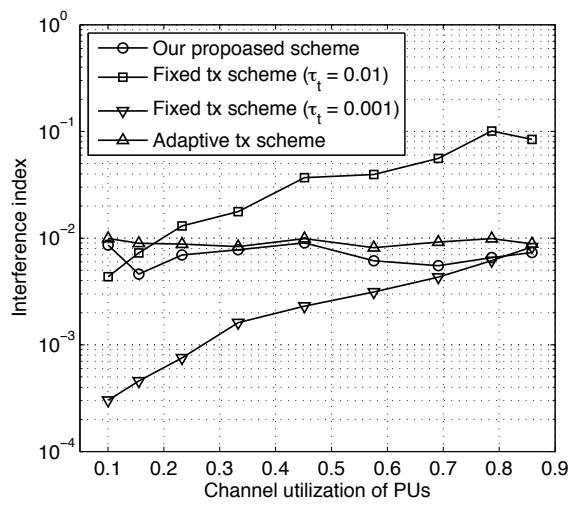

(f)

Fig. 6. The performance of our proposed scheme for different PUs' activity patterns. (a) The normalized aggregate throughput vs. the average duration of OFF state with PUs following the lognormal distribution. (b) The interference index vs. average duration of OFF state with PUs following the lognormal distribution. (c) The normalized aggregate throughput vs. channel utilization of PUs with PUs following the pareto distribution. (d) The interference index vs. channel utilization of PUs with PUs following the pareto distribution. (e) The normalized aggregate throughput vs. channel utilization of PUs with PUs following the gamma distribution. (f) The interference index vs. channel utilization of PUs with PUs following the gamma distribution.

In the rest of this section, we evaluate and compare our proposed scheme with the other schemes in terms of the normalized aggregate throughput of all SUs and interference index (to be defined in the following), when the PUs' activities follow the different distributions. The comparison schemes include the transmission scheme in the ideal case, interferenceconfined adaptive transmission scheme, and the fixed transmission scheme. In particular, the ideal case represents the "omniscient" transmission scheme under which the SUs have the full knowledge of the PUs' activities. That is, in the ideal case, the SUs are assumed to know exactly when the PBS starts and stops broadcasting packets to the PUs. Thus, the throughput of the ideal case is the upper-bound throughput that the secondary network can achieve. Furthermore, in the ideal case, the SUs do not cause any interference to the primary network.

The interference-confined adaptive transmission scheme is an interweave-based spectrum sharing scheme which is modified based on the optimal spectrum sensing framework proposed in [31], under which the SUs can access the primary channel only when the PBS is sensed idle. The original scheme which was designed for the exponential PUs traffic case only. To fairly compare the scheme in [31] with our proposed scheme, we modify the scheme in [31] so that it can adaptively adjust the transmission duration while limiting the interference caused by the SUs to a given threshold for different PUs activity patterns.

The fixed transmission scheme combines the intrusive and non-intrusive spectrum sharing modes. However, unlike our proposed scheme which takes advantage of the PUs' activity pattern, the fixed transmission scheme makes the decisions only based on the channel sensing outcomes. Particularly, under the fixed transmission scheme, the SUs constantly sense the channel. If the channel is sensed idle, the SUs transmit packets to the SBS with a fixed transmission duration. If the channel is sensed busy, the SUs adopt the non-intrusive spectrum sharing mode to transmit packets. The difference between our proposed scheme and the fixed transmission scheme is that the fixed transmission scheme does not need the mandatory sensing and the fixed transmission duration depends only on a pre-defined parameter. It is clear that for the fixed transmission scheme, the larger transmission duration may lead to a higher throughput, but more severe interference imposed onto the PUs.

In the simulations, besides the normalized aggregate throughput, we also use the interference index to evaluate the performance of our proposed schemes. We define the interference index as the ratio of the amount of time that the SUs operate in the intrusive spectrum sharing mode when PUs are active to the total simulation duration. The larger 
the interference index, the more interference caused by the SUs. In the simulations, we consider the interference occurring only when the noise level of the PU receivers is above the acceptable level due to the interference caused by the SUs that operate under the intrusive spectrum sharing mode.

First, we assume the primary network's activity pattern follows the lognormal distribution. The average ON state of the primary network lasts $0.2 \mathrm{~s}$, while the average OFF state duration of the primary network varies between $0.2 \mathrm{~s}$ and $2.8 \mathrm{~s}$. Fig. 6(a) shows the normalized aggregate throughput achieved by our proposed scheme, the fixed transmission scheme with $\tau_{t}=0.01 \mathrm{~s}$, the fixed transmission scheme with $\tau_{t}=0.005 \mathrm{~s}$, the interference confined adaptive transmission scheme with interference level of $10^{-3}$, and the transmission scheme for the ideal case. As shown in Fig. 6(a), when the duration of OFF state increases, the normalized throughput of all schemes increase. In Fig. 6(a), our proposed scheme is the scheme that achieves the closest throughput to the ideal case's throughput among all the schemes. The throughput difference between our proposed scheme and the interference-confined adaptive transmission scheme decreases as the average duration of OFF state increase. This is because our proposed scheme can get more spectrum opportunities using the non-intrusive spectrum sharing mode when the licensed channel is in ON state.

Figure 6(b) shows the interference index for the different schemes. From Fig. 6(b), we can observe that for the fixed transmission schemes, the larger the fixed transmission duration $\tau_{t}$, the larger the interference index. Our proposed scheme achieve the lowest interference index for any given channel utilization of PUs. Also, the interference index of the fixed transmission scheme increases more quickly than that of our proposed scheme when the average duration of ON state decreases. Based on Figs. 6(a) and (b), our proposed scheme significantly outperforms the fixed transmission scheme and the interference-confined adaptive transmission scheme when the PUs' activity follows the lognormal distribution.

Second, we focus on the case where the PUs' activity follows the pareto distribution. The average $\mathrm{ON}$ state duration of the primary network is $0.45 \mathrm{~s}$, while the average OFF state duration of the primary network changes between $0.025 \mathrm{~s}$ and $6.1 \mathrm{~s}$. Fig. 6(c) shows the normalized aggregate throughput of our proposed scheme, the fixed transmission scheme with different $\tau_{t}$ 's, the interference-confined adaptive transmission scheme with interference level of $10^{-3}$, and the ideal transmission scheme. The throughput of all schemes decreases as the channel utilization of PUs increases. This is because as the channel utilization of PUs increases, there is less room for the SUs to operate in the intrusive spectrum sharing mode which yields a higher throughput than the non-intrusive spectrum sharing mode. When the fixed transmission scheme adopts $\tau_{t}=0.01 \mathrm{~s}$, the throughput of the fixed transmission scheme is close to that of our proposed scheme. However, the interference caused by the fixed transmission scheme is much higher than our proposed scheme. Also, the throughput of our proposed scheme yields a much higher throughput than the interference-confined adaptive transmission scheme. Figs. 6(c) and (d) show that when the PUs' activity follows the pareto distribution, our proposed scheme can achieve better throughput and lower interference index than the fixed trans-

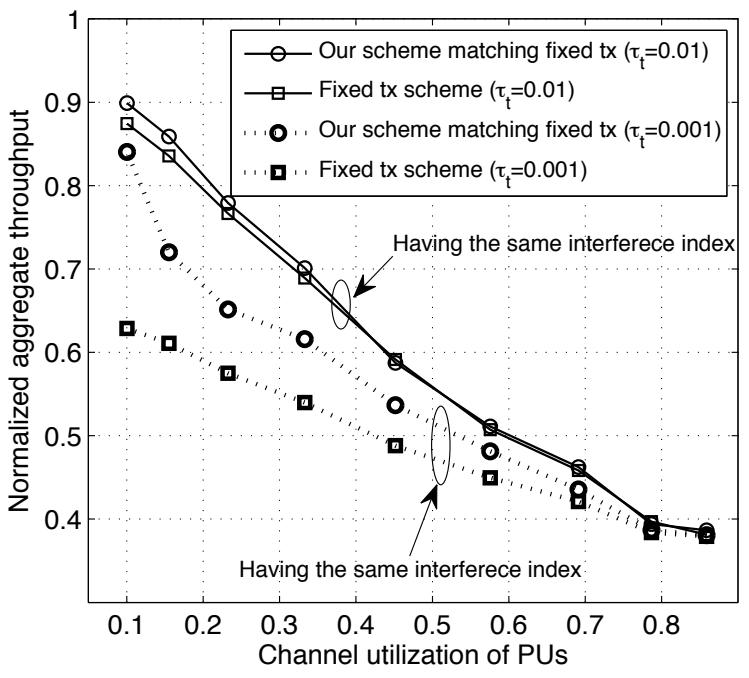

Fig. 7. Given the same interference index, the normalized aggregate throughput comparison between our proposed scheme and the fixed transmission scheme when the PUs' activity follows gamma distribution. Note that in the above figure, the plots with the same line style represent that the corresponding schemes have the same interference index.

mission scheme as well as the interference-confined adaptive transmission scheme.

Third, we evaluate our proposed scheme when the PUs' activity follows the gamma distribution. The average ON state duration of the primary network is $0.12 \mathrm{~s}$, while the average OFF state duration of the primary network changes between $0.0044 \mathrm{~s}$ and $1.1 \mathrm{~s}$. Figs. 6(e) and (f) plot the normalized aggregate throughput and interference index, respectively, against the channel utilization of PUs. The interferenceconfined adaptive transmission scheme with interference level of $10^{-2}$ achieves the lower throughput than our proposed scheme. Moreover, the interference-confined adaptive transmission scheme decreases dramatically as the channel utilization of increases because it cannot utilize the spectrum opportunities when the primary network is active. For the fixed transmission scheme, larger $\tau_{t}$ leads to a higher throughput. As shown in Fig. 6(e), the throughput of the fixed transmission scheme with $\tau_{t}=0.01$ is larger that of the fixed transmission scheme with $\tau_{t}=0.001$. When the channel utilization of PUs is less than 0.33 , our proposed scheme achieves the higher throughput than the fixed transmission scheme with $\tau_{t}=0.01$. Meanwhile, when the channel utilization of PUs is higher than 0.33 , our proposed scheme achieves lower throughput as compared to the fixed transmission scheme with $\tau_{t}=0.01$. However, we can observe in Fig. 6(f) that the interference index of our proposed scheme is much lower than that of the fixed transmission scheme with $\tau_{t}=0.01$.

It is worth noticing that the throughputs of the fixed transmission scheme and our proposed scheme converge together as the channel utilization of PUs increases when the PUs' activity follows the gamma distribution. The reason for this is twofold. On one hand, when the channel utilization of PUs is small, the optimal $\tau_{t}^{*}$ changes in a large-scale manner because the range of $\tau_{f}$ is stochastically wide. The fixed transmission scheme does not perform well because of the fixed transmission duration. On the other hand, when the channel utilization of 
PUs is large, the optimal $\tau_{t}^{*}$ changes slowly with the duration of the elapsed OFF period $\tau_{f}$, which implies that for a given $D_{t h}$, our proposed scheme may virtually choose a constant $\tau_{t}$ due to $\tau_{f}$ falling into a small range. As a result, our proposed scheme performs similarly as the fixed transmission scheme.

Thus, we further evaluate our proposed scheme as follows. For a given channel utilization of PUs, we adjust $D_{t h}$ for our proposed scheme to make our proposed scheme and the fixed transmission scheme to achieve the same interference index. Then, given the same interference index, we compare the normalized aggregate throughput of our proposed scheme with that of the fixed transmission scheme for $\tau_{t}=0.01$ and $\tau_{t}=0.001$, respectively, which is shown in Fig. 7 . When the fixed transmission scheme selects $\tau_{t}=0.001$, the improvement of our proposed scheme over the fixed transmission scheme is significant. This is because the fixed transmission scheme with smaller $\tau_{t}$ results in larger sensing overhead. In addition, when the channel utilization of PUs is small, we can see that given the same interference index, the throughput improvement of our proposed scheme over the fixed transmission scheme is noticeable. When the channel utilization of PUs keeps on increasing, the throughput of our proposed scheme converges to the throughput of the fixed transmission scheme.

\section{COnClusions}

We proposed the opportunistic spectrum sharing schemes in the CDMA-based uplink MAC over the cognitive radio networks to achieve the tradeoff between the interference imposed onto the PUs and overall spectrum utilization. In particular, we formulated the power control optimization problems for the intrusive and non-intrusive spectrum sharing modes. We designed the selection rules of intrusive and non-intrusive spectrum sharing modes. Under our proposed schemes, the SUs can dynamically determine to choose between the intrusive spectrum sharing mode and non-intrusive spectrum sharing mode based on the channel utilization, the number of active SUs, and the interference constraints. Our proposed schemes take into consideration the joint channel sensing and data transmission, and the power and rate allocations. We conducted the extensive simulations with different types of PUs' activities. Comparing with the other schemes, the simulation results show that our proposed scheme can optimally trade off the spectrum utilization with the interference caused by SUs to significantly increase the aggregate throughput of all the SUs in the cognitive radio network.

\section{APPENDIX A}

The Derivations of THE Closed-Form EXPRESSIONS FOR EQ. (27) AND EQ. (33) UNDER THE DIFFERENT

\section{DISTRIBUTIONS}

\section{1). The Lognormal Distribution:}

If the duration of the OFF period follows the lognormal distribution, then we get its cdf as

$$
C_{\tau_{0}}(t)=\frac{1}{2}\left[1+\operatorname{erf}\left(\frac{\log (t)-\mu}{\sqrt{2} \sigma}\right)\right]
$$

and its pdf as

$$
A_{\tau_{0}}(t)=\frac{1}{t \sqrt{2 \pi \sigma^{2}}} e^{-\frac{[\log (t)-\mu]^{2}}{2 \sigma^{2}}}
$$

where $\mu$ and $\sigma$ are the mean and standard deviation, respectively, in the form of natural logarithm, and $\operatorname{erf}(x) \triangleq$ $(2 / \sqrt{\pi}) \int_{0}^{x} e^{-t^{2}} d t$ is the error function. Plugging Eqs. (39) and (40) into Eq. (27), we get Eq. (41). Likewise, for the mean squared estimation error of $t_{s p}$, if the duration of the $\mathrm{ON}$ period also follows the lognormal distribution, we can obtain Eq. (42) by substituting $C_{\tau_{1}}(t)$ and $A_{\tau_{1}}(t)$, which have the same expressions as those on the righthand sides of Eqs. (39) and (40), respectively, into Eq. (33).

\section{2). The Gamma Distribution:}

If the duration of the OFF period follows the gamma distribution, then we have its cdf as

$$
C_{\tau_{0}}(t)=1-\frac{\Gamma\left(\alpha, \frac{t}{\beta}\right)}{\Gamma(\alpha)}
$$

and we get its pdf as

$$
A_{\tau_{0}}(t)=t^{\alpha-1} e^{-\frac{t}{\beta}} \frac{1}{\Gamma(\alpha) \beta^{\alpha}}
$$

where $\alpha$ and $\beta$ are the shape and scale parameters, respectively, $\Gamma(a, z) \triangleq \int_{z}^{\infty} t^{a-1} e^{-t} d t$ is the upper incomplete gamma function, and $\Gamma(a) \triangleq \int_{0}^{\infty} t^{a-1} e^{-t} d t$ is the gamma function. For the average interference duration, after plugging Eqs. (43) and (44) into Eq. (27) and algebraic manipulation,

$$
\begin{gathered}
D\left(\tau_{t}, \tau_{f}\right)=\frac{2}{1-\operatorname{erf}\left(\frac{\log \left(\tau_{f}\right)-\mu}{\sqrt{2} \sigma}\right)}\left\{\frac{\tau_{t}+\tau_{f}}{2}\left[\operatorname{erf}\left(\frac{\log \left(\tau_{t}+\tau_{f}\right)-\mu}{\sqrt{2} \sigma}\right)-\operatorname{erf}\left(\frac{\log \left(\tau_{f}\right)-\mu}{\sqrt{2} \sigma}\right)\right]\right. \\
\left.-\frac{e^{\mu+\frac{\sigma^{2}}{2}}}{2}\left[\operatorname{erf}\left(\frac{\mu+\sigma^{2}-\log \left(\tau_{f}\right)}{\sqrt{2} \sigma}\right)-\operatorname{erf}\left(\frac{\mu+\sigma^{2}-\log \left(\tau_{f}+\tau_{t}\right)}{\sqrt{2} \sigma}\right)\right]\right\} \\
\epsilon\left(t_{s p}, \tau_{i}\right)=\sqrt{\frac{2}{\pi}} e^{\frac{\mu^{2}}{2 \sigma^{2}}}\left(t_{s p}-t_{s p}^{\prime}\right)^{2}+2 \sqrt{\frac{2}{\pi} e^{\frac{\left(\mu+\sigma^{2}\right)^{2}}{2 \sigma^{2}}}\left(t_{s p}-t_{s p}^{\prime}\right)\left[\frac{\operatorname{erf}\left(\frac{\log \left(\tau_{l s}+\tau_{i}\right)-\mu-\sigma^{2}}{\sqrt{2} \sigma}\right)-\operatorname{erf}\left(\frac{\log \left(\tau_{l s}\right)-\mu-\sigma^{2}}{\sqrt{2} \sigma}\right)}{\operatorname{erf}\left(\frac{\log \left(\tau_{l s}+\tau_{i}\right)-\mu}{\sqrt{2} \sigma}\right)-\operatorname{erf}\left(\frac{\log \left(\tau_{l s}\right)-\mu}{\sqrt{2} \sigma}\right)}\right]} \\
-\sqrt{\frac{2}{\pi}} e^{\frac{\left(\mu+\sigma^{2}\right)^{2}}{2 \sigma^{2}}}\left[\frac{\operatorname{erf}\left(\frac{\log \left(\tau_{l s}+\tau_{i}\right)-\mu-2 \sigma^{2}}{\sqrt{2} \sigma}\right)-\operatorname{erf}\left(\frac{\log \left(\tau_{l s}\right)-\mu-2 \sigma^{2}}{\sqrt{2} \sigma}\right)}{\operatorname{erf}\left(\frac{\log \left(\tau_{l s}+\tau_{i}\right)-\mu}{\sqrt{2} \sigma}\right)-\operatorname{erf}\left(\frac{\log \left(\tau_{l s}\right)-\mu}{\sqrt{2} \sigma}\right)}\right]
\end{gathered}
$$




$$
\begin{gathered}
D\left(\tau_{t}, \tau_{f}\right)=\frac{1}{\Gamma\left(\alpha, \frac{\tau_{f}}{\beta}\right)}\left\{\left(\tau_{t}+\tau_{f}\right)\left[\Gamma\left(\alpha, \frac{\tau_{f}}{\beta}\right)-\Gamma\left(\alpha, \frac{\tau_{f}+\tau_{t}}{\beta}\right)\right]-\frac{\beta}{\Gamma(\alpha)}\left[\Gamma\left(\alpha+1, \frac{\tau_{f}}{\beta}\right)-\Gamma\left(\alpha+1, \frac{\tau_{f}+\tau_{t}}{\beta}\right)\right]\right\} \\
\epsilon\left(t_{s p}, \tau_{i}\right)=\left(t_{s p}-t_{s p}^{\prime}\right)^{2}+\frac{\beta}{\Gamma\left(\alpha, \frac{\tau_{l s}}{\beta}\right)-\Gamma\left(\alpha, \frac{\tau_{l s}+\tau_{i}}{\beta}\right)}\left\{\left[\Gamma\left(2+\alpha, \frac{\tau_{l s}}{\beta}\right)-\Gamma\left(2+\alpha, \frac{\tau_{l s}+\tau_{i}}{\beta}\right)\right]\right. \\
\left.+2\left(t_{s p}-t_{s p}^{\prime}\right)\left[\Gamma\left(1+\alpha, \frac{\tau_{l s}}{\beta}\right)-\Gamma\left(1+\alpha, \frac{\tau_{l s}+\tau_{i}}{\beta}\right)\right]\right\}
\end{gathered}
$$

$$
D\left(\tau_{t}, \tau_{f}\right)= \begin{cases}\left(\tau_{f}+\tau_{t}\right)\left[1-\frac{1}{1-\alpha}\left(\frac{\tau_{f}}{\tau_{f}+\tau_{t}}\right)^{\alpha}\right]+\frac{\alpha}{1-\alpha} \tau_{f}, & \tau_{f}>k \\ \left(\tau_{f}+\tau_{t}\right)\left[1-\left(\frac{k}{\tau_{f}+\tau_{t}}\right)^{\alpha}\right]-\alpha k^{\alpha} \frac{\left(\tau_{f}+\tau_{t}\right)^{1-\alpha}-k^{1-\alpha}}{1-\alpha}, & \tau_{f}+\tau_{t}>k \text { and } \tau_{f}<k \\ 0, & \text { otherwise }\end{cases}
$$

$$
\epsilon\left(t_{s p}, \tau_{i}\right)= \begin{cases}\left(t_{s p}-t_{s p}^{\prime}\right)^{2}\left(b^{\alpha}-a^{\alpha}\right)-\frac{2 \alpha(\alpha-2)\left(t_{s p}-t_{s p}^{\prime}\right)\left(b^{\alpha} a-a^{\alpha} b\right)}{\left(\alpha^{2}-3 \alpha+2\right)\left(b^{\alpha}-a^{\alpha}\right)}+\frac{\alpha(\alpha-1)\left(b^{\alpha} a^{2}-a^{\alpha} b^{2}\right)}{\left(\alpha^{2}-3 \alpha+2\right)\left(b^{\alpha}-a^{\alpha}\right)}, \quad b>k \\ 0, & \text { otherwise }\end{cases}
$$

we obtain Eq. (45). Likewise, for the mean squared estimation error of $t_{s p}$, if the duration of the ON period also follows the gamma distribution, we can obtain Eq. (46) by substituting $C_{\tau_{1}}(t)$ and $A_{\tau_{1}}(t)$, which have the same expressions as those on the righthand sides of Eqs. (43) and (44), respectively, into Eq. (33)

\section{3). The Pareto Distribution:}

If the duration of the OFF period follows the pareto distribution, then we have its cdf as

$$
C_{\tau_{0}}(t)= \begin{cases}1-\left(\frac{k}{t}\right)^{\alpha}, & t>k \\ 0, & \text { otherwise }\end{cases}
$$

and we obtain its pdf as

$$
A_{\tau_{0}}(t)= \begin{cases}\frac{\alpha k^{\alpha}}{t^{\alpha+1}}, & t>k \\ 0, & \text { otherwise }\end{cases}
$$

where $k$ is the minimum possible value of the random variable, and $\alpha$ is a positive parameter. For the average interference duration, after plugging Eqs. (47) and (48) into Eq. (27), we can derive Eq. (49). Likewise, for the mean squared estimation error of $t_{s p}$, if the duration of the $\mathrm{ON}$ period also follows the gamma distribution, by substituting $C_{\tau_{1}}(t)$ and $A_{\tau_{1}}(t)$, which have the same expressions as those on the righthand sides of Eqs. (47) and (48), respectively, into Eq. (33), we can obtain Eq. (50), where $a=\max \left\{\tau_{l s}, k\right\}$ and $b=\tau_{l s}+\tau_{i}$.

\section{REFERENCES}

[1] FCC Spectrum Policy Task Force, "Report of the spectrum efficiency working group," Technical Report 02-135, Tech. Rep., 2002.

[2] A. Goldsmith, S. A. Jafar, I. Maric, and S. Srinivasa, "Breaking spectrum gridlock with cognitive radios: An information theoretic perspective," Proc. IEEE, vol. 97, no. 5, pp. 894-914, May 2009.

[3] S. Sridharan and S. Vishwanath, "On the capacity of a class of MIMO cognitive radios," IEEE J. Sel. Topics Signal Process., vol. 2, no. 1, pp. 103-117, Feb. 2008.

[4] J. Mitola III, "Cognitive radio: an integrated agent architecture for software defined radio," Ph.D. Thesis, KTH Royal Inst. Technology, Stockholm, Sweden, 2000.

[5] S. Haykin, "Cognitive radio: brain-empowered wireless communications," IEEE J. Sel. Areas Commun., vol. 23, no. 2, pp. 201-220, Feb 2005.
[6] I. Akyildiz, W. Lee, M. Vuran, and S. Mohanty, "NeXt generation/dynamic spectrum access/cognitive radio wireless networks: a survey," Elsevier Computer Networks, vol. 50, pp. 2127-2159, 2006.

[7] N. Devroye, P. Mitran, and V. Tarokh, "Limits on communications in a cognitive radio channel," IEEE Commun. Mag., vol. 44, no. 6, pp. 44-49, June 2006.

[8] A. Mishra, "A multi-channel MAC for opportunistic spectrum sharing in cognitive networks," in Proc. Military Communications Conference MILCOM 2006, 23-25 Oct. 2006, pp. 1-6.

[9] C. Cordeiro and K. Challapali, "C-MAC: A cognitive MAC protocol for multi-channel wireless networks," in Proc. 2nd IEEE International Symposium on New Frontiers in Dynamic Spectrum Access Networks (DySPAN), 17-20 April 2007, pp. 147-157.

[10] H. Su and X. Zhang, "Cross-layer based opportunistic MAC protocols for QoS provisionings over cognitive radio wireless networks," IEEE J. Sel. Areas Commun., vol. 26, no. 1, pp. 118-129, Jan. 2008.

[11] Y. Yuan, P. Bahl, R. Chandra, P. A. Chou, J. I. Ferrell, T. Moscibroda, S. Narlanka, and Y. Wu, "KNOWS: Cognitive radio networks over white spaces," in Proc. 2nd IEEE International Symposium on New Frontiers in Dynamic Spectrum Access Networks (DySPAN), 17-20 April 2007, pp. $416-427$.

[12] X. Zhang and H. Su, "CREAM-MAC: Cognitive radio-enabled multichannel MAC protocol over dynamic spectrum access networks," IEEE J. Sel. Topics Signal Process., vol. 5, no. 1, pp. 110-123, February 2011.

[13] Y. Xing, C. N. Mathur, M. A. Haleem, R. Chandramouli, and K. P. Subbalakshmi, "Dynamic spectrum access with QoS and interference temperature constraints," IEEE Trans. Mobile Comput., vol. 6, no. 4, pp. 423-433, April 2007.

[14] D. Niyato and E. Hossain, "A game-theoretic approach to competitive spectrum sharing in cognitive radio networks," in Proc. IEEE Wireless Communications and Networking Conference WCNC 2007, 11-15 March 2007, pp. 16-20.

[15] A. Elezabi, M. Kashef, M. Abdallah, and M. M. Khairy, "Cognitive interference-minimizing code assignment for underlay CDMA networks in asynchronous multipath fading channels," in IWCMC '09: Proceedings of the 2009 International Conference on Wireless Communications and Mobile Computing. ACM, 2009, pp. 1279-1283.

[16] B. Wang and D. Zhao, "Performance analysis in CDMA-based cognitive wireless networks with spectrum underlay," in Proc. IEEE GLOBECOM, 2008.

[17] A. T. Hoang and Y.-C. Liang, "A two-phase channel and power allocation scheme for cognitive radio networks," in Proc. IEEE 17th Int Personal, Indoor and Mobile Radio Communications Symp, 2006.

[18] N. B. Chang and M. Liu, "Optimal competitive algorithms for opportunistic spectrum access," IEEE J. Sel. Areas Commun., vol. 26, no. 7, pp. 1183-1192, Sep. 2008.

[19] H. Kim and K. G. Shin, "In-band spectrum sensing in cognitive radio networks: energy detection or feature detection?" in ACM MobiCom, 2008, pp. 14-25. 
[20] Y.-C. Liang, Y. Zeng, E. C. Y. Peh, and A. T. Hoang, "Sensingthroughput tradeoff for cognitive radio networks," IEEE Trans. Wireless Commun., vol. 7, no. 4, pp. 1326-1337, Apr. 2008.

[21] A. T. Hoang and Y.-C. Liang, "Adaptive scheduling of spectrum sensing periods in cognitive radio networks," in Proc. IEEE Global Telecommunications Conference (GLOBECOM), Nov. 26-30, 2007, pp. 3128-3132.

[22] G. Ganesan, Y. Li, B. Bing, and S. Li, "Spatiotemporal sensing in cognitive radio networks," IEEE J. Sel. Areas Commun., vol. 26, no. 1, pp. 5-12, Jan. 2008.

[23] S. M. Mishra, A. Sahai, and R. W. Brodersen, "Cooperative sensing among cognitive radios," in Proc. IEEE International Conference on Communications (ICC), vol. 4, June 2006, pp. 1658-1663.

[24] G. Ganesan and Y. Li, "Cooperative spectrum sensing in cognitive radio, part I: Two user networks," IEEE Trans. Wireless Commun., vol. 6, no. 6, pp. 2204-2213, Jun. 2007.

[25] M. Wellens, J. Riihijrvi, and P. Mhnen, "Spatial statistics and models of spectrum use," Elsevier Computer Communications, vol. 32, pp. 19982011, 2009.

[26] D. Chen, S. Yin, Q. Zhang, M. Liu, and S. Li, "Mining spectrum usage data: a large-scale spectrum measurement study," in ACM MobiCom, 2009, pp. 13-24.

[27] S. W. Kim, "Adaptive rate and power DS/CDMA communications in fading channels," IEEE Commun. Lett., vol. 3, no. 4, pp. 85-87, April 1999.

[28] A. Belegundu, L. Berke, and S. Patnaik, "An optimization algorithm based on the method of feasible directions," Structural and Multidisciplinary Optimization, vol. 9, no. 2, pp. 83-88, 1995.

[29] OMNeT++. [Online]. Available: http://www.omnetpp.org/

[30] R. Jain, D. Chiu, and W. Hawe, "A quantitative measure of fairness and discrimination for resource allocation in shared systems," DEC Research Report TR-301, Tech. Rep., 1984.

[31] W.-Y. Lee and I. F. Akyildiz, "Optimal spectrum sensing framework for cognitive radio networks," IEEE Trans. Wireless Commun., vol. 7 , no. 10 , pp. $3845-3857$, Oct 2008 .

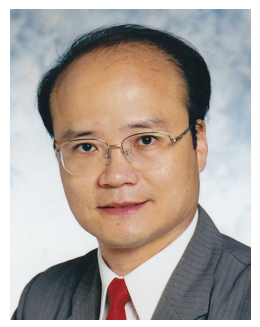

Xi Zhang (S'89-SM'98) received the B.S. and M.S. degrees from Xidian University, Xi'an, China, the M.S. degree from Lehigh University, Bethlehem, $\mathrm{PA}$, all in electrical engineering and computer science, and the Ph.D. degree in electrical engineering and computer science (Electrical EngineeringSystems) from The University of Michigan, Ann Arbor.

He is currently an Associate Professor and the Founding Director of the Networking and Information Systems Laboratory, Department of Electrical and Computer Engineering, Texas A\&M University, College Station. He was an Assistant Professor and the Founding Director of the Division of Computer Systems Engineering, Department of Electrical Engineering and Computer Science, Beijing Information Technology Engineering Institute, China, from 1984 to 1989. He was a Research Fellow with the School of Electrical Engineering, University of Technology, Sydney, Australia, and the Department of Electrical and Computer Engineering, James Cook University, Australia, under a Fellowship from the Chinese National Commission of Education. He was with the Networks and Distributed Systems Research Department, AT\&T Bell Laboratories, Murray Hills, NJ, and with AT\&T Laboratories Research, Florham Park, NJ, in 1997. He has published more than 190 research papers in the areas of wireless networks and communications systems, mobile computing, network protocol design and modeling, statistical communications, random signal processing, information theory, and control theory and systems.

Prof. Zhang received the U.S. National Science Foundation CAREER Award in 2004 for his research in the areas of mobile wireless and multicast networking and systems. He is an IEEE Communications Society Distinguished Lecturer. He received the Best Paper Awards in the IEEE WCNC 2010, IEEE GLOBECOM 2009, and the IEEE GLOBECOM 2007, respectively. He also received the TEES Select Young Faculty Award for Excellence in Research Performance from the Dwight Look College of Engineering at Texas A\&M University, College Station, in 2006.
$\mathrm{He}$ is currently serving as an Editor for the IEEE TRANSACTIONS ON COMMUNICATIONS, an Editor for the IEEE TRANSACTIONS ON WIRELESS COMMUNICATIONS, an Associate Editor for the IEEE TRANSACTIONS ON Vehicular Technology, a Guest Editor for the IEEE Journal on SELECTED AREAS IN COMMUNiCATIONS for the special issue on "Broadband Wireless Communications for High Speed Vehicles", a Guest Editor for the IEEE JOURNAL on SElECTED AREAS In COMmunications for the special issue on "Wireless Video Transmissions", an Associate Editor for the IEEE COMmunications LetTers, a Guest Editor for the IEEE Communications Magazine for the special issue on "Advances in Cooperative Wireless Networking", a Guest Editor for the IEEE Wireless Communications Magazine for the special issue on "next generation of CDMA versus OFDMA for 4G wireless applications", an Editor for the JOHN WILEY's JOURNAL ON WIRELESS COMMUNICATIONS AND MOBILE COMPUTING, an Editor for the JOURNAL OF COMPUTER SYSTEMS, NETWORKING, AND COMMUNICATIONS, an Associate Editor for the JOHN WILEY'S JOURNAL on SECURity and Communications Networks, an Area Editor for the ELSEVIER JOURNAL ON COMPUTER COMMUNICATIONS, and a Guest Editor for JOHN WILEY'S JOURNAL ON WIRELESS COMMUNICATIONS AND MOBILE COMPUTING for the special issue on "next generation wireless communications and mobile computing". He has frequently served as the Panelist on the U.S. National Science Foundation Research-Proposal Review Panels.

Prof. Zhang is serving or has served as the Technical Program Committee (TPC) Co-Chair for the IEEE INFOCOM 2013, the TPC Chair for the IEEE GLOBECOM 2011, Area TPC Chair for the IEEE INFOCOM 2012, the General Chair for IEEE ICC 2011 - Workshop on Advanced Networking Technologies for Smart-Services Based Clouding Computing, TPC Co-Chair for the IEEE ICDCS 2011 - Workshop on Data Center Performance, Panel/Demo/Poster Chairs for the ACM MobiCom 2011, TPC Vice-Chair for IEEE INFOCOM 2010, General Chair for the ACM QShine 2010, TPC Co-Chair for IEEE INFOCOM 2009 Mini-Conference, TPC CoChair for IEEE GLOBECOM 2008 - Wireless Communications Symposium, TPC Co-Chair for the IEEE ICC 2008 - Information and Network Security Symposium, Symposium Chair for IEEE/ACM International Cross-Layer Optimized Wireless Networks Symposium 2006, 2007, and 2008, respectively, the TPC Chair for IEEE/ACM IWCMC 2006, 2007, and 2008, respectively, the Demo/Poster Chair for IEEE INFOCOM 2008, the Student Travel Grants Co-Chair for IEEE INFOCOM 2007, the General Chair for ACM QShine 2010, the Panel Co-Chair for IEEE ICCCN 2007, the Poster Chair for IEEE/ACM MSWiM 2007 and IEEE QShine 2006, Executive Committee Co-Chair for QShine, the Publicity Chair for IEEE/ACM QShine 2007 and IEEE WirelessCom 2005, and the Panelist on the Cross-Layer Optimized Wireless Networks and Multimedia Communications at IEEE ICCCN 2007 and WiFi-Hotspots/WLAN and QoS Panel at IEEE QShine 2004. He has served as the TPC members for more than 90 IEEE/ACM conferences, including IEEE INFOCOM, IEEE GLOBECOM, IEEE ICC, IEEE WCNC, IEEE VTC, IEEE/ACM QShine, IEEE WoWMoM, IEEE ICCCN, etc.

Prof. Zhang is a Senior Member of the IEEE and a Member of the Association for Computing Machinery (ACM)

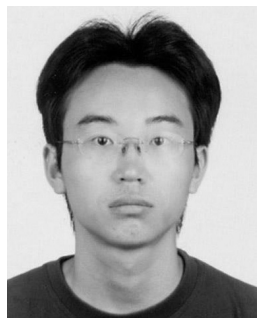

Hang Su (S'04) received B.S. and M.S. degrees, both in electrical engineering, from Zhejiang University, Hangzhou, China, in 2002 and 2005, respectively. He is currently working toward a Ph.D. degree at Department of Electrical and Computer Engineering, Texas A\&M University, College Station. He worked as a software engineer with Nokia Research Center, Hangzhou, China, in 2005. During the summer of 2009, he worked as a research intern with Mitsubishi Electric Research Laboratories, Cambridge, MA

His research interests focus on cognitive radio networks, vehicular ad hoc networks, and wireless sensor networks with emphasis on design and analysis of MAC and routing protocols. Two of his papers, both co-authored with his Ph.D. advisor Prof. Xi Zhang, received the Best Paper Awards at the IEEE GLOBECOM 2009 and the IEEE WCNC 2010, respectively.

$\mathrm{He}$ is a Student Member of the IEEE. 